\title{
INTEGRAZIONE EUROPEA E WELFARE STATES NAZIONALI
}

\author{
di Fritz W. Scharpf
}

\section{Introduzione}

Il processo di integrazione europea è caratterizzato da una asimmetria fondamentale descritta accuratamente da Joseph Weiler (1981) come un dualismo tra le norme sopranazionali europee ed il policy-making europeo di tipo intergovernativo. Weiler ha ancora ragione nella sua critica agli scienziati politici, colpevoli di aver troppo a lungo concentrato la propria attenzione soltanto sugli aspetti della negoziazione intergovernativa e di aver ignorato (o comunque non aver preso sufficientemente in considerazione) l'emergere, essenzialmente per via giurisdizionale, di un ordinamento legale europeo che prevale sulle leggi nazionali (Weiler 1994). Questa omissione è tanto più grave poiché ha impedito di individuare il parallelismo, politicamente assai significativo, tra il dualismo indicato da Weiler e la più comune contrapposizione tra una integrazione «positiva» ed una «negativa» (Tinbergen 1965; Rehbinder e Stewart 1984), cioè tra le misure che allargano l'integrazione del mercato eliminando i vincoli nazionali sugli scambi e le distorsioni competitive, da un lato, e le politiche europee comuni che modellano le condizioni sotto le quali i mercati operano, dall'altro.

Il principale beneficiario della legislazione sovranazionale europea è stata l'integrazione negativa. Le sue regole fondamentali erano già contenute nella «fonte primaria» dei Trattati di Roma. Da quel momento la liberalizzazione poté essere estesa, senza eccessiva attenzione politica, attraverso gli interventi della Commissione europea contro le violazioni degli obblighi previsti dal Trattato, ed anche attraverso le decisioni ed i pareri della Corte di Giustizia europea. L'integrazione positiva invece dipende dagli accordi tra i governi nazionali all'interno del Consi- 
glio dei ministri ed è dunque soggetta a tutti gli ostacoli propri del policy-making intergovernativo.

Questa fondamentale differenza istituzionale è sufficiente per spiegare l'asimmetria, frequentemente deplorata, tra integrazione positiva e negativa nel policy-making comunitario (Kapteyn 1991; Merkel 1993). Il risultato più probabile di ciò è un gap di competenza, in forza del quale le politiche nazionali sono fortemente limitate nella loro capacità di problem-solving, mentre le politiche europee sono vincolate dalla mancanza di accordo tra i governi nazionali. Nella misura in cui questo è vero, la political economy delle democrazie capitalistiche, quale si era sviluppata nell'Europa occidentale nelle prime decadi del dopoguerra, è stata modificata in modo fondamentale.

\section{L'integrazione negativa: la perdita di controllo sulle frontiere}

Nella storia del capitalismo, i decenni successivi alla seconda guerra mondiale sono stati caratterizzati da una insolitamente alta coincidenza tra i confini dello stato territoriale e quelli dei mercati dei capitali, dei beni, dei servizi e del lavoro'.

I confini erano certamente tutt'altro che impermeabili, ma le transazioni attraverso di essi erano comunque fortemente controllate dai governi nazionali. In conseguenza di ciò, i detentori di capitale erano solitamente disincentivati dall'investire fuori dai confini nazionali, e le aziende venivano sfidate per lo più da competitori interni. Il commercio internazionale cresceva

1 Il periodo precedente alla prima guerra mondiale ed anche gli anni venti furono fasi storiche caratterizzate dall'apertura dei mercati di capitali, dal libero commercio internazionale e dalla tendenza verso la crisi del capitalismo (Polany 1957). Nei primi anni ' 30 le principali potenze industriali risposero al problema della grande depressione con strategie protezionistiche $o$ anche autarchiche basate sulla svalutazione, sul controllo dell'esportazione di capitali, su restrizioni delle importazioni e su sussidi alle esportazioni. Il risultato di tali strategie fu il collasso dell'economia mondiale. Dopo la seconda guerra mondiale, furono necessari più di due decenni di negoziati GATT per ri-liberalizzare gradualmente il commercio internazionale, e ci vollero i due shock petroliferi prima che il mercato mondiale di capitali fosse di nuovo libero dal controllo nazionale. In retrospettiva, questa transizione graduale da economie chiuse ad una economia mondiale libera sembra aver fornito le condizioni ideali per soluzioni di tipo «social-democratico» al livello nazionale. Fino alla metà degli anni '70, in ogni modo, le società dell'Europa occidentale poterono trarre profitto dal dinamismo economico del capitalismo stabilizzando le proprie fluttuazioni attraverso un controllo macro-economico keinesiano, e correggendo le ineguaglianze distributive grazie al potere dei sindacati ed alle politiche sociali di welfare (Ruggie 1982; 1995). 
molto lentamente, e poiché i governi nazionali controllavano i tassi di cambio e le importazioni, la competitività internazionale non rappresentava un grosso problema. Finché perdurarono queste condizioni la politica dei tassi d'interesse dei governi controllava il rendimento degli investimenti finanziari. Se i tassi d'interesse venivano abbassati, gli investimenti reali che creavano lavoro sarebbero divenuti relativamente più attraenti e viceversa. Così, il controllo keynesiano poteva facilitare il ciclo economico e prevenire la disoccupazione, mentre la politica sindacale dei salari, impiegata nell'ambito di finalità macro-economiche, era in grado di controllare il tasso di inflazione. Allo stesso tempo, l'attività di regolazione del governo e la contrattazione collettiva dei sindacati controllavano le condizioni di produzione. $E$, poiché a tutti i competitori collettivi era in ultima analisi richiesto di produrre alle stesse condizioni, i costi della regolazione potevano essere trasferiti ai consumatori. Perciò, i tassi di profitto degli investimenti non erano necessariamente influenzati da alti livelli di regolazione e di potere sindacale ${ }^{2}$; l'accumulazione capitalistica fu possibile sia nel sistema di welfare svedese, dominato dai sindacati, che nel sistema di libera impresa degli USA.

Durante questo periodo, dunque, i paesi industriali dell'Europa occidentale hanno avuto la possibilità di sviluppare versioni specificatamente nazionali del welfare state capitalistico, e in effetti le loro scelte si rivelarono piuttosto differenti (EspingAndersen 1990). Al di là delle considerevoli differenze tra la versione «social-democratica», quella «corporativa» e quella «liberale» del welfare state, in tutti i casi, tuttavia, si ottenne un risultato evidente in termini di mantenimento e di promozione di una vigorosa economia capitalistica, e allo stesso tempo nel controllo, sia pure in modi diversi ed a differenti livelli, delle tendenze distruttive di un capitalismo selvaggio, assicurando in questo modo la tutela di specifici valori sociali, culturali e/o ambientali (Scharpf 1991a; Merkel 1993). Tuttavia non era sta-

2 Nella letteratura di economia politica di orientamento neo-marxista molti contributi utilizzano il declino della quota di profitto nei decenni del dopoguerra come un indicatore dell'irrisolvibile contraddizione tra l'economia capitalistica e lo stato democratico. Ma poiché l'investimento si interrompe quando il tasso di profitto del capitale diventa negativo, i governi e i sindacati dovrebbero essere consci dei rischi di una compressione dei profitti per l'occupazione e la crescita e le economie con strutture istituzionali neo-corporative sono teoricamente - e lo furono nella pratica - in grado di evitare e correggere tale errore strategico (Wallerstein 1990; Scharpf 1991a). 
to pienamente compreso in quel periodo quanto il successo delle politiche di correzione del mercato dipendesse di fatto dalla capacità dello stato territoriale di controllare i propri confini economici. Una volta perduta questa capacità, attraverso la globalizzazione dei mercati di capitale e l'integrazione trans-nazionale dei mercati di beni e servizi, gli «anni d'oro» del welfare state capitalistico sono giunti al termine.

Oggi il tasso minimo di rendimento che gli investitori possono attendersi è determinato dai mercati finanziari globali, più che dalle politiche monetarie nazionali, ed i tassi reali di interesse sono generalmente il doppio rispetto a quelli degli anni '60. Così, se un governo dovesse oggi cercare di ridurre i tassi di interesse al di sotto del livello internazionale, il risultato non sarebbe più un aumento degli investimenti reali, capaci di creare lavoro nell'economia nazionale, ma piuttosto la fuga di capitali, la svalutazione e l'aumento del tasso di inflazione ${ }^{3}$. Similmente, una volta che lo stato territoriale ha perso, o ha ceduto la sua capacità di controllo sui confini dei mercati di beni e servizi, non può più essere certo che tutti i fornitori in competizione saranno soggetti allo stesso regime regolativo. Oggi dunque, se i costi della regolazione o della contrattazione collettiva sono aumentati a livello nazionale, non possono più essere trasferiti sui consumatori. Invece, si avrà come risultato l'aumento delle importazioni, la diminuzione delle esportazioni, l'abbassamento dei profitti ed il declino degli investimenti, mentre le aziende dovranno affrontare la bancarotta o spostare la propria produzione verso luoghi più favorevoli ${ }^{4}$.

Dunque, quando il controllo sui confini declina, diminuisce anche la capacità dei governi nazionali e dei sindacati di deter-

3 Per converso, la politica monetaria nazionale ha il potere di attrarre i capitali portando i tassi di interesse al di sopra del livello internazionale. Nel fare ciò, tuttavia, si aumenta il tasso di cambio, cosa che determina una riduzione della competitività internazionale dell'economia.

4 In teoria i costi possono essere riversati sui consumatori attraverso la svalutazione della divisa nazionale. Tuttavia, gli assetti regolativi e salariali tendono ad influire solo su specifici settori dell'industria, più che sull'economia globale. La perdita di competitività non può essere così generalizzata da essere compensata (dal punto di vista dell'industria che ne è colpita) dall'aggiustamento del tasso di cambio. Oltretutto, alle condizioni di speculazione valutaria globale oggi esistenti, la competitività nelle esportazioni non è più il fattore principale nella determinazione dei tassi di cambio. Infine, una banca centrale indipendente il cui fine primario è la stabilità dei prezzi, è perfettamente in grado di stabilizzare il tasso di cambio ad un livello più alto di quanto sarebbe giustificato dalla competitività internazionale dell'economia interna. 
minare le condizioni nell'ambito delle quali può operare l'economia capitalistica. Invece, i paesi sono costretti ad una competizione per la conquista di vantaggi posizionali che ha le caratteristiche del dilemma del prigioniero (Sinn 1994). L'esempio paradigmatico di questa forma di «competizione regolativa» è stato fornito, durante il primo trentennio del secolo, dall'incapacità degli stati «progressisti» all'interno degli USA di regolare l'impiego dei bambini nell'industria. Secondo le sentenze della Corte Suprema sulla negative commerce clause, a tali stati non era permesso di proibire o di tassare $\mathrm{i}$ beni prodotti dal lavoro infantile negli stati vicini. In conseguenza di ciò, la competizione posizionale nel mercato integrato americano impediva ad uno stato di introdurre norme regolative che avrebbero influito soltanto sulle imprese dello stato stesso (Graebner 1977). Allo stesso modo, la crescente integrazione trans-nazionale dei mercati di beni e servizi, e in special modo il completamento del mercato interno europeo, riducono la libertà dei governi nazionali e dei sindacati di alzare i costi regolativi e salariali delle imprese nazionali al di sopra del livello prevalente nei luoghi in competizione. Inoltre, la «competizione dei sistemi regolativi» che è generalmente vista con favore dagli economisti e dai politici neo-liberali (Streit e Mussler 1995), può facilmente trasformarsi in una spirale negativa di deregolazione competitiva in forza della quale tutti i paesi in lizza si troveranno a dover ridurre i livelli di protezione, che risulteranno ben al di sotto di quelli preferiti da ognuno di essi.

Naturalmente le cose potrebbero cambiare, come illustrato di nuovo dall'esempio del lavoro infantile. Negli Stati Uniti è stato alla fine possibile - dopo la «rivoluzione costituzionale» del 1937 - risolvere tale problema attraverso la legislazione a livello federale. Similmente, in Europa c'è la speranza, almeno tra i sindacati ed i partiti politici ad essi legati, che ciò che è stato perduto sul piano della capacità regolativa nazionale possa essere recuperato attraverso la regolazione sociale a livello europeo. Contro queste speranze, tuttavia, si erge l'asimmetria istituzionale tra integrazione negativa e positiva, già menzionata in precedenza.

In astratto, la desiderabilità dell'integrazione negativa, o liberalizzazione, non è seriamente messa in questione dagli stati membri dell'Unione. L'impegno fondamentale di creare un «mercato comune» era certamente condiviso dai governi che siglarono i trattati comunitari e dai parlamenti nazionali che li ra- 
tificarono. Questo impegno ha trovato la sua espressione legale nelle norme fondamentali contenute nei trattati che imponevano l'eliminazione delle barriere tariffarie e non tariffarie al commercio e la realizzazione di un sistema di competizione non distorta. Ciò che probabilmente non era stato previsto all'inizio furono le dottrine sull'efficacia diretta e sulla supremazia della legge europea, presto affermate dalle sentenze della Corte Europea di Giustizia. Spiegare perché i governi nazionali abbiano accettato queste decisioni è in se stesso un tema di notevole interesse per mettere alla prova i vari approcci che studiano la teoria dell'integrazione ${ }^{5}$. In questo contesto, comunque, tale spiegazione è meno interessante degli effetti della loro acquiescenza. Una volta accettata l'efficacia diretta e la supremazia del diritto europeo, la Commissione e la Corte di Giustizia hanno avuto la possibilità di espandere continuamente l'ambito dell'integrazione negativa senza bisogno di coinvolgere il Consiglio dei Ministri'. Invece, secondo il compromesso di Lussemburgo del 1966, le misure di integrazione positiva hanno potuto essere bloccate nel Consiglio dal veto di un singolo governo.

Il significato politico-economico di questa asimmetria costituzionale diviene chiaro quando questa è paragonata alla situazione riscontrabile nell'ambito delle costituzioni nazionali. An-

5 Garrett $(1992 ; 1995)$ interpreta il caso della legge della Corte Europea di Giustizia in un quadro «intergovernativo» come il punto focale di un consenso latente tra $\mathrm{i}$ governi, mentre Burley e Mattli (1993) puntano l'attenzione sull'esistenza di un serio conflitto di interesse. Nella loro interpretazione («neofunzionalista») viene enfatizzata la relativa autonomia del sistema giuridico e la sua efficacia come uno «scudo» contro il diretto intervento politico. Sul punto cfr. anche Weiler $(1993 ; 1994)$ e Mattli e Slaughter (1995). Ciò che Garrett sembra ignorare, all'interno del suo stesso quadro di riferimento teorico, è l'importanza delle regole di decisione istituzionali: la Corte (e la Commissione in qualche settore decisionale) è capace di imporre outcomes che non avrebbero una maggioranza qualificata nel Consiglio dei Ministri, ma che non possono essere corrette dal Consiglio fino a quando i governi oppositori non sono in grado di mobilitare una contro-maggioranza qualificata in Consiglio (o quando la decisione della Corte comporta una interpretazione del Trattato, azione che richiede addirittura l'unanimità).

6 L'integrazione negativa è stata ed è perseguita dalla Commissione in via primaria attraverso le «decisioni» e le «direttive» secondo gli artt. 89 e 90 del Trattato ed attraverso l'azione contro le inadempienze da parte dei governi nazionali degli obblighi previsti dal trattato (art. 169). Almeno allo stesso livello di importanza sul piano pratico è la diretta applicazione del diritto europeo nelle cause legali ordinarie davanti alle corti nazionali e la possibilità, secondo l'art. 177 , di pareri preliminari da parte della Corte di Giustizia in risposta a richieste di una qualsiasi corte nazionale (anche di livello minore). Ancora una volta, il Consiglio dei Ministri non è coinvolto, e i governi nazionali appariranno solitamente davanti alla corte soltanto nel ruolo di accusati. 
che nella Repubblica Federale tedesca, dove la teoria neo-liberale ha guadagnato una notevole influenza nella dottrina costituzionalistica, la concezione neo-liberale dell' «economia sociale del mercato» non implica una esaltazione unilaterale della competizione, ma è stata definita, dal suo promotore originario, come la combinazione del «principio della libertà del mercato con quello della compensazione sociale» (Müller-Armack 1956, 243). Inoltre, la Corte costituzionale tedesca ha coerentemente rifiutato di attribuire status costituzionale a qualsivoglia dottrina economica, neo-liberale o di altro tipo, insistendo invece sulla «neutralità della Legge Fondamentale in materia di politica economica». Così, la libertà economica è protetta contro l'intervento statale soltanto nell'ambito del quadro generale dei diritti umani e civili, e le finalità della politica di competizione non hanno uno status costituzionale più alto degli altri legittimi fini delle politiche pubbliche. Coerentemente con questo, le misure che promuovono il mercato e quelle che lo correggono sono, in linea di principio, egualmente legittimate e, come testimoniano le vicende alterne della legislazione e della pratica anti-monopolistica, hanno entrambe da fronteggiare le stesse difficoltà nel trovare sostegno politico in un sistema fortemente pluralistico. Ciò è vero anche negli altri stati membri della Comunità europea, dove, in linea generale, le politiche pubbliche sono anche meno vincolate da dottrine relative al tipo di «costituzione economica».

Non si può ricavare dal testo dei Trattati di Roma, né dai suoi lavori preparatori, che la Comunità dovesse implicare l'abolizione di questa parità costituzionale tra la protezione della libertà economica e gli interventi per la correzione del mercato (Verloren van Themaat 1987; Joerges 1991 e 1994a; Groeben 1992).

Ciò nonostante, attraverso la supremazia del diritto europeo, le quattro libertà economiche e le ingiunzioni contro le distorsioni della competizione hanno di fatto guadagnato un valore costituzionale nei confronti degli stati membri (Mestmäker 1994, 270), mentre le opzioni corrispondenti a favore dell'intervento sociale ed economico (che al livello nazionale conterebbero sullo stesso sostegno) sono impedite dall'alto grado di consenso richiesto per l'integrazione positiva al livello europeo ${ }^{7}$.

7 Secondo le teorie neo-liberali la Comunità sarebbe stata concepita solo come un'entità che stabilisce e salvaguarda i postulati della libertà economica e della piena 
L'integrazione positiva: $i$ limiti della prospettiva intergovernativa

Mentre l'integrazione negativa è stata promossa dalla Commissione e dalla Corte Europea, se così si può dire, alle spalle dei processi politici, le misure di integrazione positiva richiedono una esplicita legittimazione politica. Fino a quando è stato applicato il compromesso di Lussemburgo, la legittimazione democratica indiretta poteva derivare dall'accordo necessario tra i governi nazionali all'interno del Consiglio dei Ministri. Il prezzo dell'unanimità comportava, naturalmente, un processo decisionale estremamente faticoso.

Si era ritenuto che l'Atto Unico Europeo del 1986 avesse cambiato questo stato di cose passando, per quelle decisioni «che hanno per oggetto la creazione ed il funzionamento del mercato interno» (art. 100A), alla regola della maggioranza qualificata in seno al Consiglio. Come conseguenza, il processo decisionale è stato di fatto accelerato, poiché oggi non è più necessario contrattare fino all'ultimo voto (Dehousse e Weiler 1990). Tuttavia, la distribuzione delle forze e le regole di voto nel Consiglio sono organizzate in modo tale che i gruppi di nazioni unite $\mathrm{da}$ interessi comuni possano difficilmente essere messi in minoranza. Così il bisogno di un consenso allargato rimane piuttosto elevato per le decisioni di integrazione positiva.

Ciò nonostante, la Comunità sta attivamente armonizzando le regolazioni nazionali in aree quali la sanità e la sicurezza industriale, i rischi ambientali, la protezione dei consumatori (Joerges 1994b; Majone 1993), e di fatto ha cominciato ad agire in questo senso anche prima dell'Atto Unico Europeo (Rehbinder e Stewart 1984). E anche dimostrato che queste regolazioni stabiliscono livelli elevati di protezione in molte aree (Eichener 1993; Voelzkow 1993; Héritier et al. 1994). Come possono queste scoperte essere riconciliate con l'affermazione precedente

competizione nell'ambito del mercato europeo. Per questo motivo l'espansione del mandato europeo, portata dal trattato di Maastricht in campi come quello della protezione ambientale, della politica industriale o della coesione sociale, è vista in modo molto critico dagli autori che appartengono a questa scuola (Mestmäker 1992; Behrens 1994). Al fine di minimizzare il danno potenziale, oggi si postula anche che «i diritti degli individui, garantiti a suo tempo dal trattato delle Comunità Europee, di partecipare al commercio oltre i confini nazionali non devono essere intaccati dai provvedimenti al servizio delle nuove competenze determinatesi» (Mestmäker 1994, 286). Se questo fosse accettato, i vincoli all'integrazione positiva non sarebbero soltanto di natura politica, ma anche costituzionale. 
secondo la quale l'integrazione positiva sarebbe impedita dalla necessità di un largo consenso nel Consiglio dei Ministri?

Al fine di risolvere questo apparente enigma è necessario esaminare la costellazione di interessi che si determina tra i governi nell'ambito del Consiglio dei Ministri ${ }^{8}$. Le regole di voto che impongono l'unanimità o anche la maggioranza qualificata istituzionalizzano di fatto le posizioni di veto, ed è giusto, sul piano analitico, affermare che, ceteris paribus, l'esistenza di una molteplicità di posizioni di veto riduce le capacità di azione politica (Tsebelis 1995). Ma se questo produrrà realmente situazioni di stallo dipende essenzialmente dalle reali costellazioni di interessi tra i partecipanti. Se tali interessi risultano essere in armonia (pure coordination games), o almeno parzialmente coincidenti (mixed-motive games), l'accordo unanime è in linea di principio possibile e potranno essere raggiunte soluzioni efficaci a dispetto della necessità di alti livelli di consenso. Situazioni di stallo sono da prevedere soltanto in presenza di costellazioni di interessi in conflitto, ma anche in questo caso l'accordo può essere raggiunto se gli sconfitti vengono compensati con pagamenti collaterali o con package deals (Scharpf 1992b). Così, se l'integrazione positiva in ambito europeo incontrerà barriere insormontabili, la spiegazione più verosimile sarà da ricercarsi nei conflitti di interesse tra gli stati membri, troppo intensi per essere appianati all'interno del quadro istituzionale dell'Unione Europea.

Conflitti di tal genere esistono certamente, ma non sono generalizzati, e non c'è ragione per pensare che siano sempre così virulenti nelle aree che dal punto di vista sostanziale e procedurale possono essere definite come quelle dell'integrazione positiva. Per dimostrare ciò, mi concentrerò sulle politiche regolative della Comunità (trascurando in questo modo i campi della politica estera e della politica di sicurezza, della giustizia e degli

8 Mi limiterò qui alla forma più semplice di spiegazione «intergovernativa». $\grave{E}$ vero naturalmente, come è stato osservato da molti critici, che i reali modelli di interazione sono più complicati. Oltre ai governi nazionali (o i Ministri specializzati rappresentati nei consigli specialistici) bisognerebbe includere perlomeno la Commissione «sovra-nazionale», le organizzazioni di interessi «sub-nazionali» e le imprese come attori di giochi complessi. Non sottovaluterei neanche la possibilità che, al fine di spiegare decisioni specifiche, modelli di giochi a due livelli e forse ancor più complessi possano venire impiegati. Pragmaticamente, comunque, ha senso, inizialmente, utilizzare il potere esplicativo dei modelli più semplici e trasparenti, aggiungendo complicazioni ulteriori solo quando necessario. In ogni modo, l'accordo tra i governi nazionali nel Consiglio dei Ministri rimane lo snodo critico per spiegare i processi decisionali comunitari. 
interni, della politica agricola comune, di quella industriale e tecnologica o i fondi sociali).

Senza entrare per il momento nelle differenze ideologiche, si può generalmente assumere che i governi nazionali mossi razionalmente dal proprio interesse considerino tre criteri di valutazione delle regolazioni proposte al livello europeo: 1) la misura in cui il contenuto della regolazione si accorda ovvero contrasta rispetto alla routine amministrativa stabilizzata a livello nazionale; 2) l'impatto verosimile sulla competitività delle industrie nazionali e sull'occupazione nell'economia nazionale, e 3) le domande specifiche e le aspettative dei propri elettorati nazionali, qualora questi siano stati attivati politicamente.

L'importanza eccezionale dei costi che ci si possono aspettare dall'aggiustamento amministrativo e procedurale nelle nazioni che sono chiamate ad attivare le regolazioni, è stata identificata nelle analisi di Adrienne Héritier e dei suoi collaboratori (Héritier et al. 1994). Queste spiegano i conflitti anche tra le nazioni che hanno un interesse comune a mantenere alti livelli di protezione regolativa. Comunque, se poi si raggiunge un accordo, è improbabile che questo riduca i livelli di protezione esistenti ${ }^{9}$. Nelle pagine seguenti mi concentrerò pertanto sui conflitti circa gli interessi economici e politici ${ }^{10}$.

La distinzione tra costellazioni di interessi consensuali ovvero conflittuali può essere grosso modo fatta corrispondere a quella tra azioni regolative centrate sul prodotto e quelle centrate sui processi produttivi (Rehbinder e Stewart 1984, 10).

9 Héritier interpreta questi conflitti come una competizione regolativa, dove alcuni paesi «ad alta regolazione» cercano di influenzare il tono della regolazione europea al fine di ridurre i propri costi di adattamento. Nel presente contesto è utile osservare che non si tratta della «competizione tra sistemi regolativi» (simile al dilemma del prigioniero), il cui risultato più tipico è la deregolazione competitiva. Nei processi studiati da Héritier, tutti gli stati membri preferirebbero accordi a livello europeo ad alto livello di protezione ambientale, ma essi divergono circa lo stile di regolazione che la Comunità dovrebbe adottare. Così, la loro competizione rassomiglia al gioco della «battaglia dei sessi» che discuteremo più avanti.

10 Analisi differenziate sono possibili, e potrebbero essere indispensabili nello studio di casi specifici. Nell'area della politica ambientale, per esempio, i governi dei paesi sviluppati economicamente e ad alto rischio di impatto ambientale devono rispondere alle pressioni incrociate degli interessi dell'occupazione nel settore industriale e di quelli degli elettori sensibili al problema ecologico. In paesi meno sviluppati, al contrario, gli interessi dei lavoratori possono essere rafforzati dalla resistenza dei consumatori ad aumenti dei prezzi causati da regolazioni ambientali stringenti. D'altra parte, naturalmente, le risposte dei governi dovranno anch'esse dipendere dalla relativa importanza delle industrie interessate nel paese in questione. 
Nel caso di regolazioni centrate sul prodotto, il mantenimento di requisiti di qualità e di sicurezza nazionali e diversificati perpetuerebbe proprio quella frammentazione dei mercati europei che i Trattati di Roma e l'Atto Unico Europeo avevano voluto superare.

Poiché tutti i paesi erano stati concordi sulla creazione di un mercato unico, si può anche assumere che l'interesse economico comune alla creazione di standard europei omogenei prevalga su altri interessi divergenti. Così, anche quando i paesi sono in disaccordo nelle loro preferenze sostanziali e procedurali, l'accordo sugli standard comuni è verosimilmente ottenibile. Questo non è vero per le regolazioni in materia ambientale e di sicurezza ${ }^{11}$ ed è ancor meno vero per regolazioni sociali del processo produttivo (Leibfried e Pierson 1992; Lange 1992). Poiché queste politiche regolative aumentano il costo della produzione, una politica nazionale diventa sempre più difficile in condizioni di competizione internazionale. $\mathrm{E}$ in questo campo, quindi, che le aspirazioni «social-democratiche» ad una ri-regolazione a livello europeo sembrerebbero essere più pertinenti. $\mathrm{Ma}$ è anche vero che qui i conflitti economici di interessi tra gli stati membri possono essere molto acuti. Al fine di giustificare questa asserzione, sarà utile un'analisi più precisa delle costellazioni di interessi in gioco.

Nel caso di regolazioni focalizzate sulla produzione, la costellazione di interessi è determinata dal quadro istituzionale: secondo l'art. 30 del Trattato le «restrizioni quantitative sulle importazioni e tutte le altre misure aventi un effetto equivalente» sono proibite tra gli stati membri. Secondo l'art. 36, comunque, tali misure sono permesse se «giustificate in base a ragioni di pubblica moralità, di politica pubblica o di pubblica sicurezza» o per «la protezione della salute e della vita di persone, animali e piante...». In altre parole, se delle regole nazionali dovessero perseguire uno dei propositi enunciati dall'art. 36, il risultato naturale, in assenza di un comune regime europeo, sarebbe la continuazione della frammentazione dei mercati in Europa. Assumendo che tutti i paesi siano orientati ad evitare tale pro-

11 Streeck $(1993,10)$ osserva giustamente che le regolazioni in materia ambientale e di sicurezza centrate sui processi produttivi possono creare ostacoli al commercio nel mercato delle macchine utensili e degli impianti di produzione. Per questa ragione, egli include tali regolazioni nella sua definizione di regole «creatrici di mercato», da distinguersi rispetto a quelle «correttive del mercato». 
spettiva, essi tuttavia si differenzieranno relativamente al livello di regolazione europea comune al quale aspirano. I paesi ricchi preferiranno generalmente livelli di protezione del consumatore e dell'ambiente più alti rispetto a quelli proposti dai paesi più poveri. Così, la costellazione risultante ricorda in qualche misura il gioco della «battaglia dei sessi» (Battle of sexes game), un gioco nel quale un accordo negoziato è generalmente difficile da ottenere, anche se non impossibile ${ }^{12}$ (figura 1).

Inoltre, anche nel caso in cui le regolazioni europee siano state armonizzate, l'art. 100A (4) dà ai paesi che preferiscono livelli più alti di protezione la possibilità di introdurre norme nazionali che applicano standard ancor più rigidi di quelli comunitari. Questo cambia il risultato naturale del gioco a favore dei paesi orientati ad un livello di regolazione più forte ed accresce il loro potere contrattuale nelle negoziazioni circa gli standard comuni. In questo modo è dunque plausibile che l'armonizzazione delle regolazioni centrate sui prodotti possa nei fatti ottenere quell'«alto livello di protezione» che l'articolo 100A (3) prevedeva nel caso della «protezione della salute e della sicurezza, dell'ambiente e del consumatore» (Eichener 1993).

Per le regolazioni centrate sui processi, tuttavia, il quadro istituzionale e le costellazioni di interessi sono molto differenti. Tali regolazioni non toccano l'utilizzazione, la sicurezza o la qualità dei prodotti realizzati in un dato modo. L'acciaio prodotto da fornaci con elevate emissioni di diossido di zolfo non è distinguibile da quello prodotto con i più dispendiosi controlli di emissione, e lo stesso è vero per le automobili prodotte dagli operai con permessi per malattie pagati o meno. Di conseguenza non è possibile in alcun modo, né in base all'art. 36 né attraverso un'altra via d'uscita contenuta nei Trattati, giustificare l'esclusione, la tassazione, o una qualsiasi discriminazione, di prodotti ottenuti sotto condizioni diverse da quelle prevalenti negli stati importatori.

Proprio come nel caso dell'esempio del lavoro infantile in America, l'ovvia implicazione è che nell'assenza di una regolazione comune a livello europeo, tutti gli stati membri si trove-

12 Inoltre, la standardizzazione relativa ai prodotti trae vantaggio dalle innovazioni procedurali che minimizzano il bisogno di consenso in sede di Consiglio dei Ministri, restringendo le sue decisioni alla sola sfera dei principi relativi alla sicurezza, mentre la specificazione dei dettagli viene lasciata alle commissioni «corporative» che rappresentano le industrie interessate e le organizzazioni nazionali per la standardizzazione (Voelzkow 1993; Eichener 1993; Scharpf 1994). 


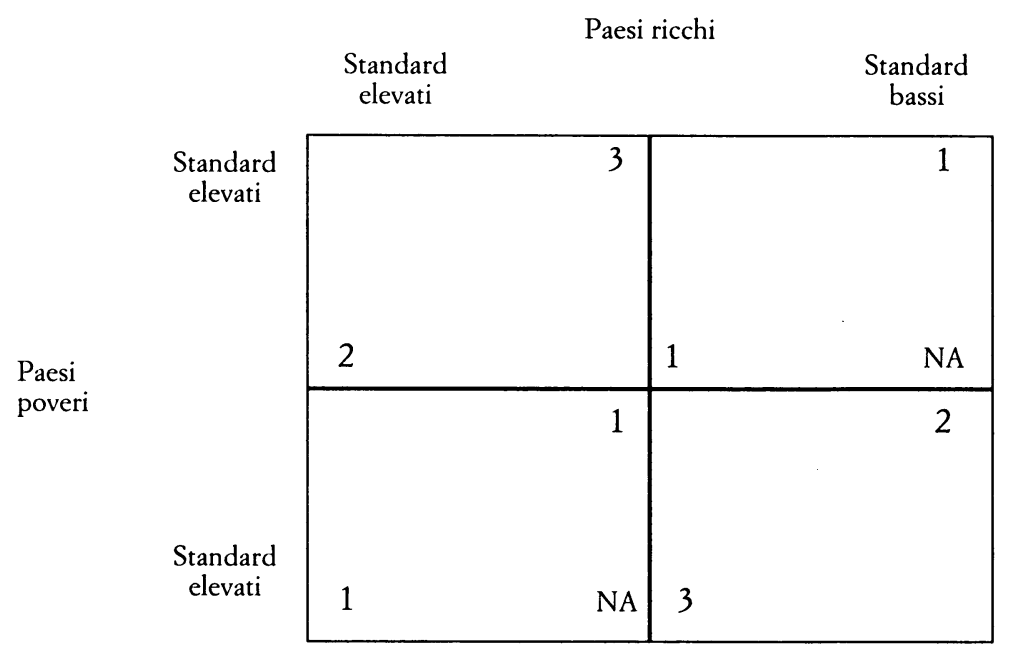

FIG. 1. Preferenze per standard europei elevati o bassi di regolazione centrata sui prodotti. In caso di «nessun accordo» (NA), nessuno standard comune viene adottato.

rebbero in una costellazione del tipo «dilemma del prigioniero», nella quale ognuno cercherebbe di ridurre le misure regolative relative ai processi e di tagliare il welfare state, al fine di migliorare la propria posizione competitiva. Di per sé questo faciliterebbe certamente l'adozione di standard comuni europei, piuttosto che impedirla. Il dilemma del prigioniero perde il suo carattere dannoso quando sono possibili accordi vincolanti tra i giocatori, e poiché questo è possibile nella Comunità europea, la ri-regolazione al livello desiderato dagli stati membri dovrebbe essere assolutamente possibile. Tuttavia è proprio qui che cominciano le difficoltà.

Esistono, in primo luogo, differenze negli stili regolativi nazionali come quelle scoperte da Adrienne Héritier et al. (1994) nel campo delle norme in materia di qualità dell'aria. Come menzionato in precedenza, questo costituirebbe un gioco del tipo «battaglia dei sessi» che si sovrapporrebbe ad uno del tipo «dilemma del prigioniero» ${ }^{13}$, il che, di per sé, non escluderebbe

13 Heckathorn e Maser (1987) hanno definito questa costellazione, nella quale una soluzione di tipo cooperativo al dilemma del prigioniero richiede un accordo su una sola delle molteplici opzioni che differiscono nelle loro caratteristiche distributive, come un «dilemma del prigioniero diviso». 
la possibilità di un accordo. Difficoltà più grandi nascono sulla base dell'esistenza di differenze ideologiche manifeste. Alcuni governi possono non condividere le preferenze «social-democratiche» e «verdi» a favore di alti livelli di regolazione, ed accettano di buon grado pressioni esterne per ottenere una de-regolazione che difficilmente potrebbero far accettare in ambito nazionale. $\mathrm{Ma}$, poiché queste difficoltà possono cambiare da un'elezione all'altra, non saranno oggetto di ulteriore investigazione in questa sede. Ciò che invece difficilmente cambia nell'alternarsi delle fasi elettorali sono i conflitti di interesse che nascono dai differenti livelli di sviluppo economico ${ }^{14}$.

Dopo il suo allargamento nel sud del continente, la Comunità europea include adesso alcuni degli stati con le economie più avanzate del mondo ed altri membri con economie di livello assai inferiore. Questo contrasto si manifesta anche in termini di notevoli differenze della produttività media ${ }^{15}$. Così, se i paesi economicamente meno sviluppati vogliono rimanere competitivi nel mercato interno europeo, $\mathrm{i}$ loro costi dei fattori produttivi, in particolare quello dei salari, nonché i costi non salariali e quelli ambientali, devono essere corrispondentemente altrettanto bassi. In effetti, i costi del lavoro industriale in Portogallo ed in Grecia sono, rispettivamente, un sesto ed un quarto di quelli riscontrabili in Germania ${ }^{16}$, e le differenze nei livelli di sviluppo del sistema di sicurezza sociale (Sieber 1993; Ganslandt 1993) e nei costi ambientali (Fröhlich 1992) mostrano gli stessi rapporti di grandezza.

Ora, se questi costi fossero alzati al livello delle nazioni più produttive, attraverso l'armonizzazione del welfare sociale e delle regolazioni ambientali, la competitività internazionale delle economie con produttività più bassa sarebbe distrutta. D'altra

14 Nella loro discussione riguardo alla politica ambientale, Rehbinder e Stewart $(1984,9)$ si concentrano invece sulla distinzione tra «stati inquinatori» e «stati ambientalisti». Tale distinzione sembra essere meno utile come spiegazione del comportamento di voto a Bruxelles, poiché gli stati altamente sviluppati producono più inquinamento $\mathrm{e}$ mostrano anche un maggiore interesse all'adozione di più stringenti regolazioni ambientali a livello europeo.

15 Naturalmente Portogallo e Grecia - esattamente come la Germania ex comunista (Hank 1994) - hanno anche isole di produttività superiori alla media, specialmente nei nuovi impianti dei gruppi multinazionali.

16 Secondo i sondaggi condotti dall'associazione degli imprenditori svedesi (SAF), i costi totali di una «ora-uomo» nell'industria spaziano, nel 1993, dalle 33 corone svedesi in Portogallo, alle 56 in Grecia alle 204 in Germania (Kosonen 1994). 
parte, se si consentisse ai tassi di cambio di scendere in misura corrispondente, si avrebbe un rialzo vertiginoso dei prezzi interni e, quindi, un impoverimento dei consumatori. Se invece i tassi di cambio fossero invariati (per esempio all'interno di una unione monetaria), il risultato sarebbe la de-industrializzazione e la massiccia perdita di posti di lavoro, proprio quanto abbiamo osservato nella Germania orientale nel momento in cui la vecchia economia socialista è stata assoggettata all'intero corpus di regolamentazioni della Germania occidentale nel contesto di una moneta unica. Più le imprese sono soggette alla competizione internazionale dei prezzi ${ }^{17}$, meno disposti saranno i politici soggetti alla responsabilità democratica dei paesi meno avanzati ad accettare le iniziative di armonizzazione che porterebbero all'incremento dei $\operatorname{costi}^{18}$. E questo è particolarmente vero al livello europeo poiché - diversamente dalla relazione tra Germania est e Germania ovest - i paesi più ricchi della comunità non saranno certo disponibili (e forse nemmeno in grado) a compensare le vittime di una simile catastrofe industriale attraverso massicci trasferimenti di denaro.

Del resto l'accordo non sarebbe più facile se il costo delle regolazioni sociali o ambientali non fosse riversato sulle imprese, ma finanziato attraverso tasse sul consumo. Visto che il reddito medio nei paesi più poveri della Comunità europea ammonta a meno di un quinto del reddito medio dei paesi più avanzati, i primi dovranno difendersi contro l'armonizzazione europea delle regolazioni ambientali o di welfare ai livelli di protezione che possono forse riflettere le aspirazioni e la volontà di pagare dei cittadini dei paesi ricchi, ma che sono al di là delle possibilità delle nazioni più povere. $\mathrm{E}$, diversamente dalla Germania orientale nel processo di unificazione nazionale tedesca, queste nazioni sono pienamente coscienti del proprio interesse, e la costituzione di una Unione europea fornisce loro un potere di veto effettivo. La costellazione che ne risulta è rappresentabile con una matrice di gioco come nella figura 2.

17 Naturalmente l'intensità della competizione dei prezzi varia a seconda dei settori. Per esempio nell'agricoltura $\mathrm{i}$ «prodotti meridionali» difficilmente competono con quelli «settentrionali».

18 Così, non è soltanto l'opposizione delle imprese che blocca il cammino della politica sociale europea (Streeck 1993). Gli stessi governi dei paesi economicamente più deboli devono, nel loro stesso interesse, anticipare e cercare di evitare l'opzione di exit del capitale. 


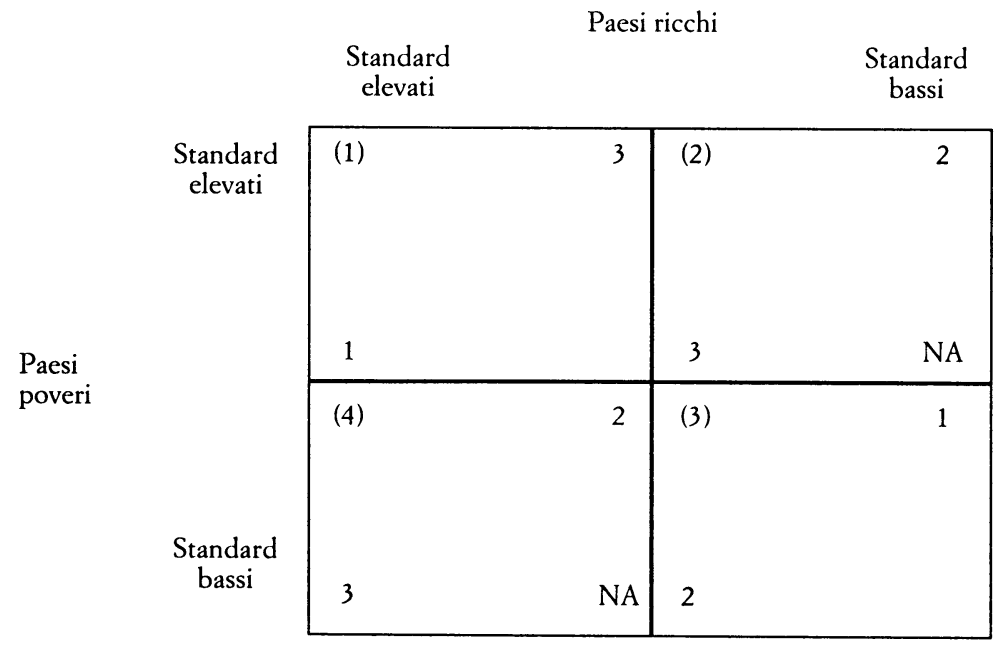

FIG. 2. Preferenze per standard europei elevati o bassi di regolazione relativa ai processi di produzione. In caso di «nessun accordo» (NA), nessuno standard comune viene adottato.

Prendiamo il caso del controllo sull'inquinamento atmosferico applicato alle emissioni industriali. Paesi ricchi, altamente industrializzati e fortemente inquinati avranno verosimilmente una chiara preferenza per standard europei ad alti livelli di protezione, come nella cella (1), in modo da proteggere anche le proprie industrie dal dumping ecologico, mentre la soluzione meno preferita sarà di avere standard comuni a bassi livelli di protezione (cella 3). Per i paesi poveri, invece, gli standard alti significherebbero la distruzione dei loro settori industriali scarsamente produttivi. $\mathrm{Ma}$ anche regole comuni che impongano standard bassi in modo uniforme (cella 3) non sarebbero così attraenti, poiché le imprese locali meno produttive sarebbero esposte ad una pericolosa competizione con attori produttivi delle nazioni più ricche. Così, per loro, il miglior risultato potrebbe essere il non-accordo (celle 2 e 4 ) che costituisce anche la seconda scelta per le nazioni ricche. Dunque il risultato è che lo status quo è probabilmente destinato a rimanere ${ }^{19}$.

19 Se i settori industriali interessati non giocano un ruolo importante negli stati membri meno sviluppati, il danno causato dalle regolazioni europee ad un alto livello di protezione può essere abbastanza piccolo da essere compensato attraverso pagamenti collaterali utilizzando il fondo per la coesione. E stato anche suggerito in diverse occa- 

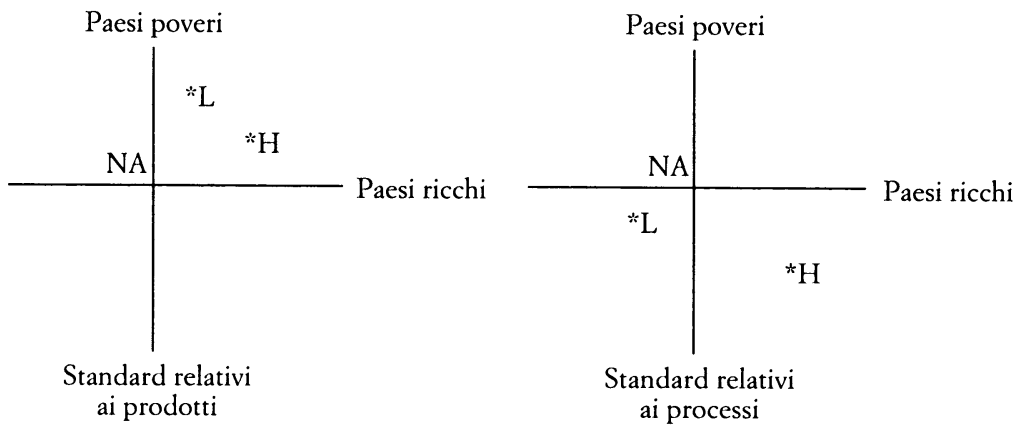

FIc. 3. Accordo negoziato circa standard europei elevati $(\mathrm{H})$ e bassi (L) confrontati con situazioni di non accordo (NA) nei due casi di standard relativi ai prodotti ed ai processi di produzione.

Le differenze tra le negoziazioni circa le regolazioni relative ai prodotti ed ai processi diventano ancora più evidenti se le opzioni sono rappresentate nella forma di un diagramma bi-dimensionale della negoziazione, nel quale le dimensioni orizzontale e verticale rappresentino l'utilità dei diversi outcomes desiderati, rispettivamente, per i paesi ricchi e per quelli poveri (fig. 3). I punti $\mathrm{H}$ e $\mathrm{L}$ rappresentano la collocazione degli accordi vincolanti rispettivamente su standard elevati e bassi. Tuttavia, poiché il punto di origine (NA) rappresenta il miglior risultato che ogni paese può ottenere quando non si raggiunge nessun accordo a livello europeo (di modo che si continuano ad applicare gli standard nazionali), lo spazio di negoziazione è in realtà limitato solo al quadrante «di nord est» in alto ed a destra del punto di origine.

Nel caso di regolazioni relative ai prodotti, i paesi ricchi preferiranno accordi su standard elevati $(\mathrm{H})$ mentre quelli poveri cercheranno di ottenere standard bassi (L). Entrambi i gruppi di paesi tuttavia preferiranno una qualsiasi soluzione al risultato di un mancato accordo (NA). Per cui entrambe le soluzioni sono collocate nello spazio di negoziazione e l'accordo su una di essa, o il compromesso tra esse, dovrebbero essere in teoria raggiungibili. Naturalmente, dovendo seguire la regola dell'unanimità, un negoziato sul problema dei vantaggi relativi ambiziose possono essere un riflesso di procedure di implementazione relativamente meno ambiziose. 
potrebbe trascinarsi e, in presenza di condizioni sfavorevoli, la contrattazione potrebbe anche fallire.

Dunque appare perfettamente razionale che i governi, nell'Atto Unico Europeo, si siano alla fine accordati per muoversi verso una forma di maggioranza qualificata specificamente sull'armonizzazione delle regolazioni relative ai prodotti (art. $100 \mathrm{~A})$. Questo permette infatti di evitare intoppi e di accelerare le negoziazioni nell'ambito di costellazioni dove generalmente si preferisce l'accordo al disaccordo.

La situazione è differente per le regolazioni relative ai processi di produzione. In questo caso non è possibile trovare una soluzione nel quadrante in alto a destra, tale da essere la preferita da entrambi i gruppi di paesi rispetto allo status quo. Dal punto di vista dei paesi poveri anche l'adozione di standard europei ad un basso livello di protezione sarebbe infatti peggiore rispetto al mantenimento dello status quo. D'altra parte i paesi ricchi preferirebbero migliorare la propria posizione introducendo standard europei di livello elevato, ma questa soluzione non può essere imposta contro la resistenza degli stati meno avanzati $^{20}$.

Per sintetizzare, l'integrazione positiva al livello europeo ha raggiunto notevoli risultati nell'armonizzazione delle norme regolative relative ai prodotti, mentre quella circa le norme regolative relative ai processi produttivi in materia ambientale e di welfare incontra molte difficoltà. D'altra parte, l'integrazione negativa sta seriamente restringendo le capacità degli stati membri di trattare i problemi generati dall'integrazione dei mercati di capitali, beni e servizi. Se questo stato di cose è da considerarsi non soddisfacente, si possono logicamente cercare soluzioni in due direzioni: o accrescendo le capacità di problem-solving al livello europeo, o proteggendo le capacità degli stati membri di svolgere un'azione efficace anche in condizioni di mercati trans-nazionali integrati.

20 Anche laddove il Trattato di Maastricht permette in via generale la procedura di voto a maggioranza qualificata sulle misure ambientali (art. 130S), cinque nazioni delle sei con i costi salariali e non salariali del lavoro più bassi nell'Unione (Portogallo, Grecia, Spagna, Irlanda, Gran Bretagna e Italia) possono facilmente formare una minoranza capace di bloccare quelle regolazioni che danneggerebbero la propria competitività. 
Prima soluzione: la crescita delle capacità europee di problem-solving

Nell'affrontare conflitti di interessi pervasivi, il problem-solving a livello europeo potrebbe essere facilitato attraverso riforme istituzionali in grado di accrescere la capacità di risolvere $i$ conflitti, o attraverso la ricerca di strategie, sostantive o procedurali capaci di ridurre la conflittualità a livelli più accettabili.

\section{a) Soluzioni maggioritarie?}

Ovviamente, la capacità di risoluzione dei conflitti sarebbe molto rafforzata se l'Unione Europea proseguisse nel cammino verso l'estensione delle procedure di voto a maggioranza assoluta all'interno del Consiglio dei Ministri, che è iniziato con l'Atto Unico Europeo e poi con il trattato di Maastricht. Se le decisioni potessero in generale essere adottate a maggioranza semplice, le nazioni ad alta produttività potrebbero, almeno nell'attuale situazione ed ammesso che esse siano capaci di accordarsi tra di loro, imporre i propri alti standard al resto della comunità. $\mathrm{Ma}$, naturalmente, i cambiamenti costituzionali nell'Unione Europea continuano a dipendere dal consenso unanime tra tutti i membri, ed il fatto che l'allargamento verso nord dell'Unione stessa sia stato sul punto di fallire proprio sul problema della procedura di voto dimostra che i potenziali perdenti sono difficilmente disposti ad accettare un regime nel quale sarebbero poi continuamente messi in minoranza.

Inoltre, se fosse possibile estendere le procedure di voto a maggioranza semplice nel Consiglio dei Ministri, il dibattito sul «deficit democratico» nell'Unione Europea si riaprirebbe con grande forza. Fintantoché la legittimità democratica del governo europeo resta essenzialmente legata all'accordo tra $\mathrm{i}$ governi nazionali, democraticamente responsabili, i cittadini dei paesi i cui governi fossero messi in minoranza nel Consiglio saranno portati a considerare tali decisioni prive di una legittimazione democratica ${ }^{21}$. Infatti, anche le caute estensioni delle procedure

21 Il quadro teorico di questa proposizione può essere qui soltanto suggerito (Scharpf 1970). Un'esigenza di legittimazione sorge quando le decisioni contrastano con le preferenze di alcune parti in causa. Fino a tempi recenti, la Comunità Europea era capace di basarsi essenzialmente su una forma di legittimità output oriented, secondo la 
di voto a maggioranza qualificata nell'Atto Unico e nel Trattato di Maastricht hanno scatenato reazioni giudiziarie e pubblici dibattiti negli stati membri, evidenziando critiche così profonde alla legittimità dell'attuale struttura decisionale nel Consiglio, tali da far pensare che ogni ulteriore progresso debba aver bisogno di più solida fondamenta di legittimazione (Groeben 1992; Weidenfeld 1995) ${ }^{22}$.

Molti critici ritengono ancora che la soluzione più appropriata sia quella definita nel progetto costituzionale di Altiero Spinelli, che avrebbe trasformato la Comunità Europea in uno stato federale dotato di un parlamento bicamerale. La prima camera avrebbe dovuto essere l'attuale Parlamento Europeo, eletto direttamente e con pieni poteri legislativi e di bilancio, mentre il Consiglio, come seconda camera, avrebbe rappresentato gli interessi degli stati membri, nello stile del Bundesrat tedesco. La Commissione avrebbe preso invece il posto di un governo europeo, essendo eletta dal Parlamento europeo e davanti ad esso responsabile (Williams 1991; Featherstone 1994). Ciò che si frappone è, naturalmente, l'egoismo istituzionale dei governi degli stati membri che non sono disposti a rinunciare al proprio controllo sul policy-making europeo. E non è tutto; proposte come queste si basano anche su deboli fondamenta in fatto di teoria democratica.

La legittimità democratica non è, dopo tutto, una questione di pure competenze formali di un parlamento. La rappresentanza e la regola di maggioranza assicurano legittimità soltanto in un contesto caratterizzato: a) dalla preesistente identità collettiva di un corpo politico che può giustificare l'imposizione di sacrifici su alcuni membri della comunità nell'interesse dell'insieme della comunità stessa; $b$ ) dalla possibilità ${ }^{23}$ di una riflessione

quale la massimizzazione del benessere comune e un'equa allocazione di costi e benefici sono i criteri cruciali. Ma da quando gli interventi europei sono diventati più frequenti e più importanti, ed i loro effetti allocativi più visibili, la legittimità input-oriented (che coinvolge $\mathrm{i}$ problemi della giustificazione e della responsabilità democratica degli attori decisionali) ha guadagnato una salienza centrale.

22 Questo non significa rifiutare la possibilità di ogni forma di legittimazione non maggioritaria (Majone 1994a e 1994b; Dehousse 1995). Ma il rispetto dell'expertise, dell'imparzialità e della correttezza procedurale che può legittimare le decisioni della Corte, delle banche centrali, o delle commissioni regolative indipendenti sul modello americano difficilmente basterà per assicurare la legittimazione democratica degli scambi scaturiti nel Consiglio dei Ministri.

23 È stato spesso sostenuto che la Comunità europea non dovrebbe essere valutata sulla base degli standard ideali ma irrealistici di pratica democratica che vengono spesso 
pubblica su quali sacrifici debbano in effetti essere imposti, per quali scopi ed a quali soggetti; infine $c$ ) dalla responsabilità politica di leader visibili al pubblico e capaci di esercitare un potere effettivo.

Nella storia del governo democratico, queste precondizioni non sono mai state soddisfatte ad un livello superiore rispetto a quello dello stato nazionale (Calhoun 1993; Dahl 1994). Esse non sono oggi soddisfatte nell'ambito dell'Unione Europea, ed è certamente poco chiaro come esse potrebbero essere create in un futuro prossimo (Grimm 1992; Kielmansegg 1992; Scharpf 1992a e 1993). Per adesso, in ogni caso, l'identità politica e culturale dell'Unione Europea è ancora molto debole (Wilson e Smith 1993); la mancanza di una lingua comune rappresenta un grande ostacolo alla crescita di una riflessione pubblica sull'idea di Europa (Gerhards 1993) e la conseguenza di ciò è che non possiamo contare su media europei, su partiti politici europei, e su uomini politici con una visibilità ed una responsabilità di livello europeo. Queste condizioni non sono facilmente ottenibili attraverso le riforme costituzionali ${ }^{24}$, e finché non ci sarà un cambiamento a questo livello il voto a maggioranza semplice nel Parlamento Europeo non produrrà certamente l'accettazione delle decisioni in quei paesi o in quei gruppi i cui interessi siano danneggiati.

Per il momento è improbabile che le riforme istituzionali possano aumentare in modo significativo la capacità di risolu-

violati in tutti gli stati membri. La mia opinione al riguardo è che questa interpretazione non centra il vero problema. Nelle condizioni attuali, una democrazia può soltanto essere definita come potenziale. Non è possibile e neanche necessario che ogni materia sia trattata in piena luce di fronte all'opinione pubblica, purché ogni detentore di cariche pubbliche sia conscio che ogni caso potrebbe essere politicizzato. Quando ciò è assicurato, la «legge delle reazioni anticipate» garantisce il resto.

24 Secondo il mio punto di vista, ulteriori crescite delle competenze legislative del Parlamento Europeo non rappresentano la strategia più promettente nel breve periodo, poiché con tale allargamento si renderebbe il processo di decision making europeo ancora più lento e complesso di quanto non sia oggi. Invece, se il presidente della Commissione fosse eletto direttamente e responsabile pienamente di fronte al Parlamento Europeo stesso, questo aiuterebbe a concentrare l'attenzione dei media su una posizione altamente visibile della leadership politica; per ottenere ciò è però necessario che nel Parlamento Europeo i partiti presentino candidati con un forte appeal europeistico. Questo fenomeno condurrebbe anche, nel tempo dovuto, alla formazione di partiti politici europei (Weidenfeld 1995). Come osserva Dehousse (1995), tuttavia, l'introduzione di orientamenti partitici nella Commissione renderebbe piuttosto difficili i suoi rapporti con i governi nazionali presenti nel Consiglio, molto più difficili di quanto siano adesso, un argomento questo che trova evidenti riscontri nella prassi del federalismo tedesco. 
zione dei conflitti a livello europeo. Così la diagnosi di Weiler (1981) già menzionata all'inizio di questo saggio, continuerà ad essere valida: contrariamente ai processi giuridici in corso, che tendono a definire ed a far valere la legge sovra-nazionale dell'integrazione negativa, i processi politici richiesti per sviluppare l'integrazione positiva manterranno il loro carattere intergovernativo e saranno facilmente frenati nel momento in cui gli interessi nazionali divergeranno. Stando così le cose, sembra tuttavia utile esplorare anche le strategie di riduzione dei conflitti che potrebbero essere capaci di trattare efficacemente i proble$\mathrm{mi}$ che non possono più essere maneggiati al livello nazionale.

\section{b) Soluzioni basate sulla prevenzione dei conflitti}

C'è in effetti un intero gruppo di strategie di questo genere (Scharpf 1994). Una di esse è stata già menzionata in precedenza. Nell'armonizzazione degli standard relativi ai prodotti, l'accordo è stato facilitato restringendo l'azione del Consiglio alla formulazione dei «principi» e lasciando i dettagli all'elaborazione degli organi corporativi incaricati della standardizzazione. In più, nelle regolazioni in materia ambientale relative ai processi produttivi, l'art. 130T adesso permette in via generica ad ogni stato membro di mantenere o introdurre misure protettive più stringenti rispetto a quelle comunitarie, posto che esse siano «compatibili con il Trattato» (cioè con l'integrazione negativa). Così, un modo per superare il blocco descritto poc'anzi sarebbe un accordo su livelli minimi di protezione accettabili per gli stati membri meno avanzati, mentre quelli più ricchi rimarrebbero liberi di mantenere gli standard più alti già esistenti che essi considerano necessari. Ma si può considerare questa una soluzione efficace del problema? Per i paesi che avevano standard regolativi molto bassi i nuovi standard comuni potrebbero richiedere certamente un cambiamento significativo. Ma i paesi ad alto standard si troverebbero nella stessa situazione di quella «competizione tra i sistemi regolativi» che ha impedito agli stati americani nel primo terzo di questo secolo di adottare regolazioni contro il lavoro infantile.

Una soluzione parziale per questo problema fu fornita dal passaggio, in sede di Commissione, da un orientamento di ispirazione tedesco circa gli standard di emissione agli standard di qualità dell'aria del genere di quelli favoriti dal governo britan- 
nico (Héritier et al. 1994). Poiché, nell'insieme (eccezion fatta per metropoli come Atene), il problema dell'inquinamento atmosferico è tipico delle regioni maggiormente industrializzate della comunità, l'adozione di standard uniformi richiederà generalmente alle nazioni più avanzate di introdurre misure antiinquinamento più stringenti rispetto a quelle di cui necessitano i paesi meno evoluti. Così, l'impatto differenziale delle norme sulla qualità dell'aria non soltanto faciliterà l'accettazione da parte delle nazioni più povere degli standard più elevati, ma servirà anche a proteggere i paesi caratterizzati da alti livelli regolativi contro le tentazioni di una deregolazione.

$\mathrm{Ma}$, naturalmente, la circostanza fortunata per cui l'intensità dei problemi di inquinamento varia in relazione alla capacità ed alla volontà dei paesi di pagare per la loro soluzione, non si verifica in tutte le occasioni. Per esempio, non si sarebbe verificata nel caso paradigmatico delle regolazioni sul lavoro infantile. Ciò nonostante, ci deve essere una lezione generale da imparare dalle regolazioni in materia di qualità dell'aria: non è necessariamente vero che l'armonizzazione europea, al fine di essere utile, debba avere lo stesso impatto su tutti gli stati membri o su tutte le regioni della comunità.

\section{c) Regolazioni a due livelli?}

Più esattamente, ciò che vorrei mettere in rilievo è il fatto che gli ostacoli agli accordi in materia di regolazioni centrate sui processi produttivi potrebbero essere ridotti in modo consistente da una nuova versione dell'idea di «Europa a geometria variabile» che, per quanto mi è dato sapere, non è stata sinora presa in considerazione nell'attuale dibattito sulla riforma istituzionale della comunità ${ }^{25}$.

Tale suggerimento è basato sull'assunto per il quale il gioco del dilemma del prigioniero che le nazioni europee sono porta-

25 Rassegne sistematiche sulle precedenti discussioni e sulle pratiche attuali sono fornite da Nicoll (1984) e da Langeheine e Weinstock (1984). Ci sono state anche proposte per una «Europa delle relatività» che definirebbe generalmente standard europei comuni in termini di criteri sensibili alle differenze nello sviluppo economico dei vari paesi. Per esempio, gli introiti derivanti da una tassazione ambientale a livello europeo potrebbero essere stabiliti come una percentuale del prodotto interno lordo al fine di evitare pesi sproporzionati a danno degli stati membri più poveri (Weizsäcker 1989). Modelli simili sono stati discussi anche in riferimento alla politica sociale. 
te a giocare tra di loro, in presenza di una integrazione negativa e nell'assenza di una armonizzazione europea, non sia giocato con eguale intensità da tutti gli stati. La competizione tra i sistemi regolativi è verosimilmente più acuta per quei paesi che sono in diretta competizione economica perché producono gli stessi beni a livelli di produttività e di costi di produzione simili. Al contrario, le nazioni che producono a livelli molto diversi di produttività e di costi di produzione non sono generalmente in competizione sullo stesso mercato. Se questo è vero, il fallimento dell'adozione di un unico standard europeo implicherebbe che due distinti giochi del tipo dilemma del prigioniero siano giocati contestualmente: uno tra le nazioni più efficienti, capaci di competere sul piano della produttività, ed uno tra le economie meno efficienti, che invece devono competere sui costi di produzione.

La soluzione sembra allora ovvia: al fine di bloccare la pressione verso una deregolazione competitiva ${ }^{26}$, c'è sì bisogno di armonizzare le regolazioni relative ai processi produttivi a livello europeo, ma non c'è necessariamente bisogno di un unico standard comune. Invece ciò che sarebbe necessario è un esplicito accordo su due standard in grado di offrire differenti livelli di protezione a differenti livelli di costo. Le nazioni ricche potrebbero quindi impegnarsi ad adottare regolazioni con standard elevati, che corrispondono alle loro esigenze sul piano ambientale e su quello sociale, mentre i paesi meno sviluppati potrebbero stabilire standard comuni al livello inferiore, che li proteggerebbero comunque dai pericoli di una rovinosa competizione tra di loro. Seguendo il corso dello sviluppo economico, gli standard più bassi potrebbero naturalmente essere rimodulati, passo dopo passo, e riallineati con quelli più alti.

A paragone con le difficoltà che si avrebbero per raggiungere accordi tra paesi poveri e ricchi su un unico standard europeo uniforme, queste negoziazioni su un doppio standard dovrebbero essere molto più facili (fig. 4). Inoltre, al contrario di altre proposte per una Europa a due velocità, il club delle nazioni ad alta regolazione non avrebbe alcun interesse ad escludere i pretendenti tra i paesi che ritengono di potersi conforma-

26 L'integrazione negativa nella Comunità Europea comprende complicati casi di ingiunzioni contro le distorsioni di competitività create dai sussidi e dai benefici pubblici preferenziali ed altre forme di «azioni affermative» che favoriscono i produttori nazionali, ma nessuna azione contro la pratica della deregolazione competitiva. 


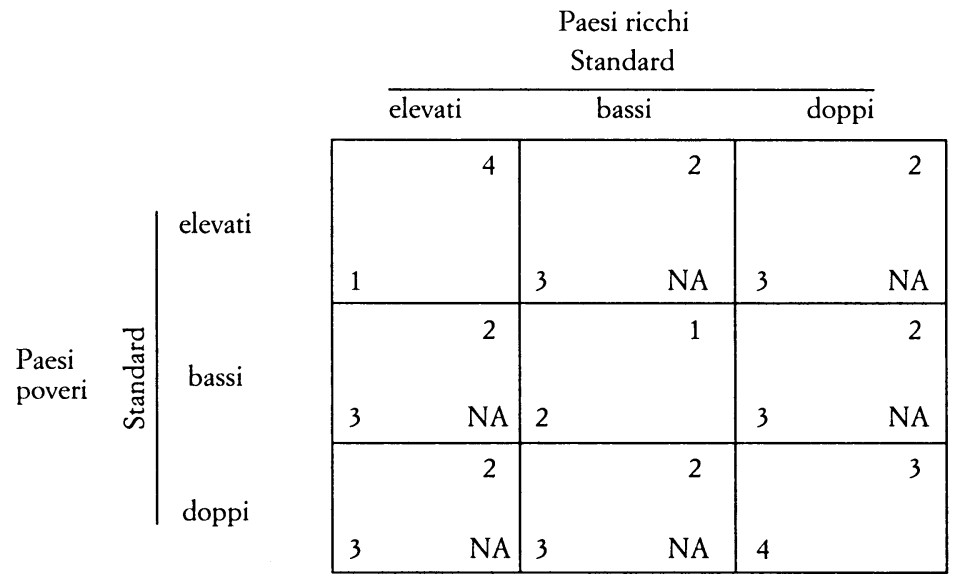

FIG. 4. Regolazioni relative ai processi con l'opzione di un doppio standard. $\mathrm{NA}=$ risultato in caso di mancato accordo.

re agli standard più elevati. La scelta più difficile sarebbe quella affrontata dai paesi della fascia media, come Gran Bretagna o Italia, che dovrebbero decidere se sia più conveniente la sfida sulla produttività o quella sui costi.

\section{d) L'importanza delle istituzioni}

Finora ci siamo concentrati soltanto sulle negoziazioni tra i paesi ricchi e quelli poveri, ed abbiamo assunto che all'interno di ognuno di questi gruppi l'accordo dovrebbe essere raggiungibile senza troppi problemi, purché le differenze tra le nazioni in termini di stile regolativo non siano troppo salienti. Ma ciò che è stato detto si applica pienamente soltanto all'armonizzazione delle regolazioni in materia ambientale relative ai processi produttivi. Nel caso di regolazioni circa le relazioni industriali o di welfare sociale, al contrario, anche l'armonizzazione a due livelli potrebbe incontrare grandi difficoltà a causa della rilevanza molto più evidente delle differenze qualitative e istituzionali. Così, mentre può essere assunto che tutti i paesi preferirebbero un ambiente meno inquinato sempreché essi se lo possano permettere, tale assunzione sulle aspirazioni comuni non può valere nel campo delle relazioni industriali ed in quello del welfare (Esping-Andersen 1990). 
Svezia e Svizzera, per esempio, sono sul piano economico tra i paesi più sviluppati del mondo, eppure differiscono in modo evidente nella quota di prodotto interno lordo che destinano ai trasferimenti ed ai servizi pubblici in materia di welfare. Oppure, la Gran Bretagna e la Germania hanno livelli molto simili di densità sindacale, ma le strutture dei loro sindacati sono molto diverse così come i sistemi di contrattazione collettiva. Ancora più importante, sotto questo aspetto, è il fatto che le relazioni industriali in Germania sono inserite dentro un sistema molto sviluppato, e controllato per vie giudiziarie, di diritto del lavoro, di norme sulla contrattazione collettiva e sulla co-determinazione; le relazioni industriali britanniche invece, sin dall'inizio di questo secolo, si sono sviluppate all'insegna del principio della «libera contrattazione collettiva» e sull'intesa per cui la legge dello stato non dovrebbe interferire nelle interazioni tra capitale e lavoro. Così, può essere vero che, al di là di ogni considerazione sui costi o sui possibili pagamenti collaterali, ogni tipo di norma regolativa circa le relazioni industriali a livello europeo sarebbe inaccettabile non soltanto per gli imprenditori, ma anche per gli stessi sindacati inglesi. Al contrario, i sindacati tedeschi, ma anche quelli austriaci o francesi, in questa materia ripongono la loro fiducia sull'efficacia legale e sull'applicazione giurisdizionale delle regolazioni statali (Crouch 1993). E, naturalmente, queste differenze istituzionali sono difese da interessi organizzati politicamente molto potenti che nessun governo può trascurare facilmente.

Nel campo del welfare sociale e delle relazioni industriali, perciò, la costellazione di interessi anche tra le nazioni ad alto livello di sviluppo economico non può essere interpretata come un gioco, di relativamente facile soluzione, sul tipo della battaglia dei sessi, come quello che abbiamo postulato nel campo delle regolazioni in materia ambientale. Invece, se assumiamo che il gruppo delle nazioni ad alto livello di regolazione include due tipi qualitativamente diversi di soluzioni istituzionali, avremo una costellazione di interessi nella quale entrambe le parti potrebbero preferire il non accordo piuttosto che un tentativo di armonizzare sistemi con differenti caratteristiche (fig. 5).

Costellazioni potenzialmente simili di interessi possono esistere in tutte quelle aree dove le differenze istituzionali tra gli stati membri hanno forte rilevanza politica, o perché esistono potenti gruppi di interesse a difesa dello status quo istituzionale, o perché le strutture istituzionali tradizionali sono diventate un 
Paesi del Tipo I

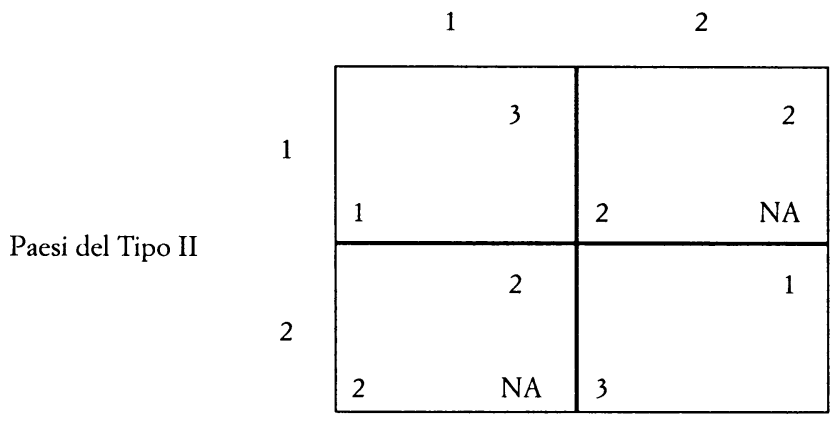

FIG. 5. Armonizzazione delle regolazioni in materia di welfare e di relazioni industriali tra paesi con livelli simili di sviluppo economico, ma con tipi differenti di istituzioni.

$\mathrm{NA}=$ risultato in caso di mancato accordo.

componente delle identità politiche e sociali. Questo è ovviamente vero per le istituzioni politiche e amministrative vere e proprie, ma lo può essere anche per le strutture istituzionali di molti altri settori che, in tutte le nazioni, sono stati protetti dallo stato, in un modo o nell'altro, contro gli effetti del mercato. Tradizionalmente nell'Europa occidentale questi settori definibili come «prossimi allo Stato» (Mayntz e Scharpf 1995) includono l'istruzione e la ricerca di base, la sanità, la radio e la televisione, le telecomunicazioni, i trasporti, le risorse energetiche e quelle idriche, la gestione dei rifiuti, i servizi finanziari, l'agricoltura e molti altri.

Si tratta di un insieme eterogeneo di settori, nei quali le giustificazioni per il coinvolgimento dello stato, così come le modalità di tale coinvolgimento, variano molto, dalla fornitura diretta di servizi ed infrastrutture attraverso agenzie statali finanziate dal fisco, ai monopoli pubblici e privati finanziati dai consumatori o altamente regolati, alle forme di auto-regolamentazione professionale sostenute dallo stato, fino alle forniture private ma sussidiate dallo stato di beni di mercato e di servizi.

Ciò che è comune a tutte queste modalità è una qualche forma di isolamento rispetto alla competizione illimitata del mercato. E ciò che conta qui è che l'attenuazione delle pressioni del mercato combinata con la varietà delle possibili forme di intervento statale ha generalmente prodotto lo sviluppo di solu- 
zioni istituzionali piuttosto diverse per quello che riguarda la fornitura di beni e di servizi simili negli stati membri dell'Unione Europea (Alber e Bernardi Schenklunn 1992).

Dal punto di vista della Comunità europea, praticamente ognuna di queste soluzioni istituzionali può essere considerata come una barriera non salariale e, certamente, come una distorsione della competizione. Così la logica dell'integrazione negativa implica che esse debbano essere rimosse, come attualmente sta accadendo in campi quali le telecomunicazioni, il trasporto aereo e i servizi finanziari. D'altra parte, non tutte queste soluzioni istituzionali restrittive e protezionistiche sono prive di una valida giustificazione, cosicché, nella logica dell'art. 36 e dell'art. 100A, o dell'art. 76, l'armonizzazione europea di questi regimi settoriali potrebbe sembrare la risposta più appropriata.

$\mathrm{Ma}$ come potrebbe il Consiglio ottenere un accordo su un sistema comune europeo di finanziamento e di gestione dei servizi sanitari in grado di sostituire le consolidate varietà di sistemi sanitari nazionali britannico, italiano e svedese, nonché i sistemi presenti in Francia, Germania ed Austria, che mescolano formule di assicurazione sanitaria obbligatoria con la fornitura privata di servizi sanitari, il tutto attraverso negoziazioni corporative tra compagnie assicurative e fornitori organizzati? In questo caso gli ostacoli all'armonizzazione saranno pesanti almeno quanto quelli presenti nel campo delle pensioni di anzianità. Il passaggio dal modello assicurativo tedesco del pay-as-you-go ad un (forse più desiderabile) sistema comune basato sul modello britannico « a doppio ordine», che combina pensioni basate sui contributi (volontari od obbligatori) con assicurazioni integrative private, risulta infatti praticamente impossibile poiché alla generazione attiva in questo momento sarebbe richiesto di pagare un prezzo doppio, uno per la presente generazione di pensionati che hanno maturato $i$ diritti previdenziali nel vecchio sistema ed uno per la loro stessa pensione nel nuovo sistema.

Un esempio particolarmente istruttivo è fornito dalla comparazione dei settori delle telecomunicazioni e dell'energia che sembrano essere abbastanza simili in molti aspetti economici (Schmidt 1995). In entrambi i settori, le strutture monopolistiche hanno prevalso fino alla metà degli anni ' 80 , e la Commissione ha lavorato da allora per la liberalizzazione in entrambe le aree. Ma mentre nel campo delle telecomunicazioni la combinazione della liberalizzazione europea con la deregolazione nazionale e la privatizzazione, ed anche caute ri-regolazioni a livello 
europeo, hanno avuto successo con una certa velocità (Sauter 1995), i tentativi ripetuti della Commissione di liberalizzare il mercato dell'energia elettrica europea sono falliti, fino a questo momento, in sede di Consiglio. Come ha mostrato Suzanne Schmidt nel suo studio comparato, uno dei due fattori più importanti che spiegano le differenti traiettorie della liberalizzazione è la differenza istituzionale (Schmidt 1995) ${ }^{27}$. Mentre nel settore delle telecomunicazioni le strutture istituzionali in tutti $\mathrm{i}$ paesi europei hanno avuto, negli anni '70, lo stesso andamento convergente verso il modello del monopolio pubblico delle poste e delle telecomunicazioni, detentore delle reti fisiche e allo stesso tempo fornitore di tutti i servizi e dell'equipaggiamento per il pubblico (Schneider 1995), il settore dell'elettricità è invece caratterizzato da un considerevole livello di eterogeneità. È vero che esistono ovunque monopoli di reti di distribuzione, ma questi monopoli possono essere a livello nazionale, regionale o locale; possono appartenere allo stato o ad investitori privati; possono essere ristretti alla generazione ed alla distribuzione di elettricità, o possono anche distribuire gas od altre forme di energia. Inoltre, ci sono considerevoli differenze nei regimi regolativi sotto i quali questi monopoli operano, e nella logica dei loro rispettivi sistemi di determinazione dei prezzi; e ci sono, naturalmente, anche differenze sostanziali nel modo in cui il conflitto circa l'energia nucleare è stato affrontato nei vari stati membri. In conseguenza, la liberalizzazione influenzerebbe $\mathrm{i}$ fornitori dei vari stati in modo piuttosto diverso, mentre l'appello a favore di una «armonizzazione precedente alla liberalizzazione» metterebbe i governi nazionali di fronte al problema ancor più difficile di dover sfidare frontalmente interessi istituzionali consolidati.

In breve, ci sono di fatto importanti settori per i quali l'armonizzazione a livello europeo dei sistemi regolativi ed istituzionali nazionali non può essere considerata una opzione possibile. La questione è, a questo punto, se l'integrazione negativa

27 L'altro fattore identificato dalla Schmidt $(1995,18)$ è la differenza nella vulnerabilità alla competizione internazionale. Nelle telecomunicazioni, se non ci fosse una liberalizzazione coordinata al livello europeo, la condizione di default che le nazioni dovrebbero fronteggiare sarebbe la scelta tra una liberalizzazione nazionale od una perdita di quote di mercato a favore di più efficienti competitori stranieri che, grazie allo sviluppo tecnologico, potrebbero invadere i mercati nazionali. Nel campo dell'elettricità, al contrario, la viabilità economica delle regolazioni nazionali ed i monopoli delle reti non sono stati toccati dal recente sviluppo tecnologico. 
debba fare il suo corso in tutti i settori nei quali le strutture istituzionali possono essere interpretate come freni al commercio o distorsioni della libera competizione. Se prevale questa impostazione, gli equilibri di valori e gli interessi oggi incorporati all'interno di istituzioni nazionali specifiche saranno ribaltati. In alcuni settori questi costi sono stati considerati accettabili sul piano politico, ma non c'è ragione per assumere che questo debba valere ovunque ${ }^{28}$. Dove questo invece non vale, l'integrazione negativa sarà vigorosamente avversata, oppure le sue conseguenze provocheranno fenomeni di disintegrazione sociale e di delegittimazione politica del tipo di quelli causati nella Germania orientale dalla rimozione delle istituzioni indigene.

\section{Seconda soluzione: ripristinare il controllo nazionale sui confini?}

Sembra utile, dunque, riflettere anche sui modi con i quali si possono porre limiti alla crescita quasi automatica, all'interno della Comunità Europea, dell'integrazione negativa, non sufficientemente meditata e motivata da considerazioni di pura efficienza economica. Questo non è un grosso problema, naturalmente, nelle aree dove la liberalizzazione deve essere ottenuta attraverso decisioni del Consiglio dei Ministri. I governi che siano seriamente intenzionati a mantenere le strutture istituzionali esistenti sono ancora in grado di bloccare le iniziative della Commissione, come è stato dimostrato proprio dai recenti fallimenti della liberalizzazione dei mercati europei nel campo dell'energia elettrica. I governi non hanno poteri formali, tuttavia, per impedire alla Commissione di procedere contro «imprese nazionali privilegiate» attraverso l'uso di direttive sulla base dell'art. 90 (3) del trattato ${ }^{29}$, ed hanno ancora meno controllo sul-

28 In Germania, per esempio, questo significherebbe che i canoni obbligatori imposti ai cittadini al fine di finanziare la televisione pubblica sarebbero contestati con successo da parte dei network privati come un sussidio che distorce la competizione, e il monopolio dell'assistenza sanitaria domiciliare da parte dei medici privati potrebbe essere intaccato dall'invasione di organizzazioni di assistenza medica in stile americano. Ora, mentre entrambi questi cambiamenti potrebbero essere considerati molto opportuni da alcuni, è altrettanto chiaro che non troverebbero il sostegno di alcuna maggioranza rappresentativa al livello nazionale.

29 D'altra parte, i governi che, per motivi interni, potrebbero non voler aderire ad una direttiva del Consiglio, possono invece preferire la deregolazione per mezzo delle direttive e delle decisioni della Commissione. 
l'uso, da parte della Commissione, del suo potere di emanare «decisioni» contro singoli governi nazionali, sulla base dello stesso art. 90, o di iniziare procedimenti davanti alla Corte, sulla base dell'art. $169^{30}$. Inoltre, data l'efficacia diretta del diritto primario europeo, ogni individuo o corporazione può sfidare le strutture istituzionali esistenti al livello nazionale di fronte ad un tribunale del proprio paese, per poi ottenere un giudizio preliminare dalla Corte di Giustizia europea, secondo i dettami dell'art. 177.

I controlli politici non funzioneranno dunque in maniera generalizzata, o meglio, funzioneranno in modo asimmetrico. Finché il Consiglio sarà tenuto a procedere attraverso maggioranze qualificate o con decisioni unanimi, una piccola minoranza sarà capace di bloccare l'azione positiva, mentre maggioranze molto larghe dovranno mobilitarsi per poter correggere l'allargamento dell'integrazione negativa prodotto dalle decisioni della Commissione ${ }^{31}$ o della Corte di Giustizia ${ }^{32}$. La questione diventa allora se sia possibile usare strumenti giuridici per limitare le capacità della Commissione e della Corte di estendere l'integrazione negativa oltre i limiti ritenuti politicamente accettabili dal Consiglio.

Nel trattato di Maastricht, è vero, i governi esclusero esplicitamente la Corte di Giustizia dalle aree della «politica estera comune e della politica di sicurezza» e da quella della «cooperazione nel campo della giustizia e degli affari interni» (Art. L). Questa è una sicura indicazione del fatto che i poteri della Corte di convertire le obbligazioni dei Trattati in diritto sovra-na-

30 Proprio nel campo dell'energia elettrica, la Commissione ha appena iniziato azioni in tal senso contro la Francia, la Danimarca, la Spagna, l'Italia, l'Irlanda e l'Olanda. Inoltre, la spinta alla liberalizzazione nel campo delle telecomunicazioni fu iniziata * in virtù di un'azione condotta con successo dalla Commissione contro la British Telecom nel 1985 (Sauter 1995).

31 Per esempio, quando la Commissione decise la sua direttiva sugli equipaggiamenti privati, secondo l'art. 90 (3), la Francia fu affiancata dall'Italia, il Belgio, la Germania e la Grecia nell'iniziativa di una (infruttuosa) azione, seguendo l'art. 173, contro alcuni punti cruciali della direttiva. Se la direttiva non fosse stata decisa in seno alla Commissione, ma fosse stata introdotta dal Consiglio secondo l'art. 100, il gruppo degli oppositori sarebbe stato sicuramente abbastanza forte da prevenire la sua adozione (Sauter 1995, 101).

32 Infatti, come sostiene Suzanne Schmidt (1995, 25 ss.), la mera possibilità di una liberalizzazione «incontrollata» tramite la Corte può persuadere i governi oppositori ad accettare una «liberalizzazione coordinata» attraverso le direttive del Consiglio, nella speranza che queste possano essere prese in considerazione nella stessa interpretazione del testo del trattato da parte della Corte. 
zionale, e di interpretare il significato di tali obbligazioni oltre l'intento originario delle parti contraenti, è divenuto finalmente materia di seria preoccupazione da parte degli stati membri. È anche possibile che preoccupazioni simili circa il ruolo della Corte possano aver contribuito all'inclusione della «clausola di sussidiarietà» nell'art. 3B (2) del trattato comunitario. In ogni caso, questo proposito può difficilmente essere raggiunto attraverso la clausola stessa (lasciando per ora stare i possibili effetti del cambiamento del clima politico che essa simboleggia).

Restringendo il principio di sussidiarietà alle «aree che non cadono nelle competenze esclusive della Comunità», la dinamica dell'integrazione negativa (che naturalmente può essere solo una competenza esclusiva europea) è rimasta completamente intatta, ma, come ho sostenuto, è proprio il campo dell'integrazione negativa quello dove la Commissione e la Corte sono capaci di esercitare il loro potere maggiore, e più pericoloso per le autonomie nazionali. Inoltre, anche riguardo all'integrazione positiva, la clausola di sussidiarietà difficilmente può avere un'efficacia giuridica molto significativa (Dehousse 1993). Data l'eterogeneità delle condizioni e delle risorse degli stati membri, è difficilmente immaginabile che un tribunale possa annullare una data misura europea, sostenuta da una maggioranza qualificata in seno al Consiglio dei Ministri, negando la validità della motivazione alla base di una tale misura cioè che «gli obbiettivi dell'azione proposta non sono ottenibili adeguatamente dagli stati membri». Così, è probabilmente più realistico ritenere che la clausola di sussidiarietà sia fondamentalmente un richiamo politico ad auto-limitarsi diretto allo stesso Consiglio dei Ministri ${ }^{33}$.

Ciò che determinerebbe invece una differenza significativa, in termini giuridici, nell'integrazione negativa ed anche in quella positiva, è quanto emerge proprio da quella sentenza che ha permesso all'integrazione negativa di fare un vero e proprio «passo da gigante». Nella sentenza sul Cassis de Dijon (120/78 ECR, 1979, p. 649), la Corte non sostenne, come è stato a volte ritenuto, che il «mutuo riconoscimento» dei prodotti autorizzati

33 Questo non sarebbe di per sé completamente privo di significato, poiché le burocrazie degli stati membri possono di fatto utilizzare le direttive europee per aggirare i controlli dei parlamenti nazionali. La stessa tendenza dei governi locali a promuovere una «sovra-integrazione» al livello centrale è stata osservata nel federalismo tedesco (Scharpf 1988). 
da altri stati membri costituisse un obbligo incondizionato per un membro della comunità. Prima che alla Germania fosse imposto di ammettere il liquore francese, la Corte aveva esaminato la tesi secondo la quale il requisito imposto in Germania di un contenuto più elevato di alcool fosse giustificabile come una norma sanitaria, trovandola del tutto falsa (Alter e Meunier-Aitsahalia 1994, 338-339). Se le cose non fossero andate così, una restrizione sull'importazione sarebbe stata ammessa, ai sensi dell'art. 36 del trattato che permette restrizioni quantitative «giustificate sul piano della pubblica moralità, delle politiche pubbliche, della pubblica sicurezza, della protezione della salute e della vita di esseri umani, di animali e di piante...» posto che tali misure «non costituiscano un mezzo di discriminazione arbitraria o di restrizioni occulte ai commerci tra gli stati membri».

Così, è il trattato stesso a riconoscere determinati obbiettivi delle politiche nazionali come capaci di prevalere sui dettami dell'integrazione dei mercati. È vero che la Commissione, ed ancora di più la Corte Europea di Giustizia, hanno fatto del loro meglio per assicurare la priorità dell'integrazione negativa applicando test piuttosto severi prima di accettare che una regolazione nazionale non sia da considerare discriminatoria o come una restrizione occulta al commercio. In effetti, la Commissione ha seguito una linea coerente, secondo la quale le regolazioni nazionali relative ai prodotti saranno o annullate, sulla base della sentenza sul Cassis, in quanto prive di valide finalità, o rimpiazzate da regolazioni armonizzate a livello europeo secondo l'art. 100A (Alter e Meunier-Aitsahalia 1994). Ciò che interessa, in questa sede, è però l'implicazione opposta: le regolazioni nazionali che restringono le importazioni e che perseguono una delle valide finalità elencate nell'art. 36 , potranno rimanere in vigore a meno che o fino a quando non subentrino armonizzazioni a livello europeo.

Circa le regolazioni relative ai prodotti, l'integrazione negativa non ha perciò precedenza su quella positiva, ed il gap di competenza menzionato in apertura di questo saggio è di fatto evitato. Tuttavia questo non è vero per quello che riguarda le regolazioni relative ai processi di produzione, che non potrebbero essere salvaguardate sulla base dell'art. 36 poiché non riguardano la qualità o la sicurezza dei prodotti stessi. Inoltre, tali regolazioni non devono violare le norme europee sulla competizione (artt. 85 e seguenti), non devono isolare le agenzie di 
servizi pubblici dalla competizione (art. 90) e non devono costituire un aiuto statale che contribuisca alla distorsione del sistema competitivo (art. 92).

E importante osservare che queste proibizioni si applicano indipendentemente dal fatto che ci siano state precedenti armonizzazioni europee oppure no. Un esempio di ciò è offerto dalla politica europea dei trasporti che, insieme all'agricoltura, è uno dei due campi nei quali il trattato originale aveva previsto un regime pienamente europeizzato (artt. 74 e seguenti). Poiché, di fronte ai grandi conflitti di interesse tra gli stati membri, il Consiglio era stato incapace di agire per oltre 25 anni, esso fu spinto dalla Corte (attraverso un procedimento promosso dal Parlamento Europeo, secondo l'art. 175) a stabilire perlomeno le condizioni per l'integrazione negativa, secondo i dettami dell'art. 75 (1) lettere (a) e (b). Inoltre, la Commissione e la Corte sono intervenute contro quelle regolazioni nazionali (come per esempio la tassa tedesca sul trasporto su strada) che potevano essere interpretate come discriminazioni per trasportatori non nazionali (art. 76). Pertanto, contro l'intento originario delle parti contraenti è in corso un'attiva liberalizzazione del mercato europeo dei trasporti anche se un accordo su un sistema regolativo europeo non è ancora in vista.

Se questo stato di cose è considerato non soddisfacente, si può immaginare la necessità di andare nella direzione indicata da norme come quelle contenute negli artt. 36, 48 (3), 56 (1), 66 e $100 \mathrm{~A}$ (4) che permettono limitazioni al libero movimento di beni, persone e servizi se tali limiti corrispondono a finalità di pubblica moralità, di politica pubblica, di sicurezza pubblica, di salute pubblica, ecc. In pratica, nessuna di queste eccezioni è a tutt'oggi di grande importanza, poiché la Commissione, ed ancor di più la Corte, le hanno interpretate in un modo estremamente restrittivo, e la stessa cosa si è verificata per altre norme stabilite nell'identica prospettiva, come l'esonero parziale dalle norme sulla competizione per le imprese nazionali relative ad infrastrutture od al risparmio (art. 90, 2) o la ricerca sui sistemi nazionali relativi alla proprietà (art. 222). In tutti questi casi, la priorità di fatto dell'integrazione negativa sulle preferenze di policy e sulle tradizioni istituzionali nazionali è stata ribadita attraverso l'interpretazione giudiziaria.

Rimane da vedere se lo stesso destino dovrà riguardare anche alcune delle più esplicite clausole limitative introdotte dal trattato di Maastricht, come per esempio quella nell'art. 126 (1) 
che permette alla Comunità un accesso solo molto limitato nella sfera dell'istruzione, «purché sia rispettata pienamente la responsabilità degli stati membri relativamente al contenuto dell'insegnamento, all'organizzazione del sistema scolastico ed alle diversità culturali e linguistiche». Con questo linguaggio, la clausola pone soltanto limiti alle competenze molto circoscritte della Comunità in materia di istruzione, ma non offre una salvaguardia contro azioni basate sul fatto che i sistemi scolastici possano rappresentare dei freni agli scambi e distorsioni della competizione nel mercato dei servizi scolastici.

Perché le preferenze nazionali di policy e le tradizioni istituzionali possano avere la possibilità di sopravvivere, sembra che siano necessari vincoli legali ancora più potenti per bloccare l'imperialismo dell'integrazione negativa. Una soluzione radicale sarebbe l'abolizione dello status di legge costituzionale europea attribuito alla normativa sulla competizione togliendola dai trattati e lasciando la determinazione della sua portata al processo politico della legislazione «secondaria» promossa dal Consiglio e dal Parlamento. Questo creerebbe, a livello europeo, un equilibrio costituzionale tra proposte di policy in competizione tra loro, come succede in tutti i sistemi politici nazionali. Inoltre, potrebbe essere esplicitamente stabilito che la legislazione nazionale rimanga in vigore finché non sia evidenziato in modo chiaro il suo contrasto con una specifica norma della legislazione europea. Questa è la regola osservata negli USA da quando, nel 1937, si abbandonò la dottrina della negative commerce clause (Schwartz 1957; Rehbinder e Stewart 1984), e questo è anche lo stato de facto del diritto comunitario relativamente alle regolazioni in materia di prodotti nel mercato dei beni. Tale regola potrebbe e dovrebbe essere estesa anche ai mercati dei servizi, e in particolare ai trasporti ed ai servizi finanziari.

Questi cambiamenti, diversamente dalla clausola di sussidiarietà, farebbero davvero la differenza, e la Conferenza Intergovernativa in preparazione per «Maastricht II» potrebbe promuoverli. Inoltre, potrebbe essere utile enumerare, nel trattato stesso, le aree di policy per le quali gli stati membri dovrebbero mantenere una responsabilità primaria. Le aree più plausibili in questo senso dovrebbero essere quelle discusse sopra, e cioè l'istruzione, la cultura, i media, il welfare sociale, l'assistenza sanitaria, le relazioni industriali e naturalmente l'organizzazione politica e amministrativa.

Come ho sostenuto in altre occasioni, questo darebbe alla 
costituzione della Comunità un carattere bi-polare, simile al «Federalismo duale» che la Corte Suprema americana aveva letto nella Costituzione degli Stati Uniti prima del 1937, o alle decisioni della Corte costituzionale tedesca che protesse la $\mathrm{Kul}$ turbobeit dei Laender nel campo dell'istruzione e in quello dei media (Scharpf 1991b e 1994; Weidenfeld 1995). Non c'è, naturalmente, alcuna speranza che possa essere definita una chiara linea di demarcazione tra le aree europee e quelle nazionali di responsabilità. $\mathrm{Ma}$ un dualismo esplicito forzerebbe la Corte e la Commissione a bilanciare le richieste di perfezionamento sul piano economico dell'integrazione nel mercato con le richieste egualmente legittime per il mantenimento dell'autonomia istituzionale e delle capacità di problem-solving nazionali alla luce delle circostanze concrete del caso specifico. Invece di decidere contro regolazioni nazionali ogni volta che venga identificata la più insignificante distorsione della competizione, la Corte dovrebbe valutare il livello di restrizione della competizione o della mobilità contro l'importanza della misura per la realizzazione dei legittimi fini degli stati membri. Ciò che viene richiesto, in altre parole, è il management dell'interdipendenza (Dehousse 1993; Joerges 1994a), in modo che si possano affrontare, nella relazione «verticale» tra competenze nazionali ed europee, quelle tensioni tra finalità economiche e non-economiche che, negli stati-nazione, sono risolte in una relazione «orizzontale» attraverso i conflitti inter-settoriali che possono riscontrarsi nel governo o in parlamento.

Ma che effetti ci sarebbero se tali cambiamenti costituzionali fossero adottati, e, una volta adottati, se avessero l'impatto desiderato sull'interpretazione giuridica dell'integrazione negativa? La Comunità europea, dopo tutto, deve mantenere l'impegno alla creazione di un mercato comune, e detenere anche strumenti legali per difendere il libero accesso ai mercati nazionali contro il protezionismo economico dei suoi stati membri. Così, i divieti contro le restrizioni quantitative sugli scambi e contro le discriminazioni nei confronti di produttori stranieri dovrebbero certamente rimanere in piedi. Ciò che potrebbe cambiare è il livello di perfezionismo con il quale essi sono andati definendosi e la loro priorità «lessicografica» rispetto ad ogni altra considerazione. Cosa ancora più importante: $\mathrm{i}$ cambiamenti costituzionali del tipo discusso sopra proteggerebbero, o ristabilirebbero, il potere dei governi nazionali di mantenere taluni settori completamente fuori del mercato o di organizzarli 
in modo da limitare gli effetti della forza di mercato. Se questo implicasse una perdita di efficienza economica, non dovrebbe spettare alla comunità evitare che gli stati membri paghino questi prezzi.

Regolazione sociale in una sola nazione?

$\mathrm{Ma}$ anche se il vestito stretto dell'integrazione negativa dovesse allentarsi e qualche settore dovesse rimanere sotto il più intenso controllo dello stato territoriale, questo non rovescerebbe automaticamente i cambiamenti fondamentali occorsi nella political economy dei welfare states capitalistici dalla fine dell'epoca post-bellica. La parte prevalente dell'economia nazionale è esposta alla competizione trans-nazionale, il capitale è diventato mobile sul piano globale, e le imprese sono in grado di riallocare la produzione attraverso tutta l'Europa Occidentale senza rischiare il loro accesso ai mercati nazionali. E poiché la mobilità dei fattori economici è cresciuta, la capacità nazionale di ridurre il tasso di profitto degli investimenti sotto il livello internazionale, sia attraverso l'abbassamento dei tassi di interessi, sia con l'imposizione di costi addizionali alle aziende, è stata irrevocabilmente persa (Sinn 1993). In questo senso, non c'è alcuna via che possa riportare indietro all'«età dell'oro» dei welfare states capitalistici del dopoguerra.

Dal punto di vista della democrazia politica, sarebbe molto dannoso negare l'esistenza di questi vincoli economici; ma sarebbe ugualmente pericoloso esagerarne il significato. $\grave{\mathrm{E}}$ vero che la capacità di una gestione macro-economica di tipo keynesiano non è più riscontrabile a livello nazionale e non è ancora possibile a livello sovra-nazionale. $\grave{E}$ anche vero che il tasso di profitto degli investimenti produttivi, che i detentori del capitale possono ottenere, è considerevolmente cresciuto. Ogni tentativo, del governo o dei sindacati, di recuperare quanto hanno perduto con programmi ridistributivi in un contesto nazionale sarebbe destinato a fallire.

$\mathrm{Al}$ di là di ciò, tuttavia, il carattere fondamentale della relazione tra economie capitalistiche e stati democratici è ancora lo stesso. Come osservavo in precedenza, anche nel periodo postbellico la regolazione sociale dell'economia capitalistica ebbe successo soltanto perché i costi della stessa, che erano in prima istanza imposti alle aziende, venivano successivamente trasferiti 
sui consumatori. In conseguenza, $i$ profitti sugli investimenti rimasero positivi, ed il capitalismo rimase ugualmente vitale nel welfare state svedese, nell'economia sociale di mercato tedesca o nel sistema di libera iniziativa degli USA. In altre parole, la simbiosi del dopoguerra tra capitalismo e democrazia poté avere successo soltanto perché alla fine i costi del welfare state furono pagati dai lavoratori e dai consumatori, più che dai detentori di capitale.

Se questa sorta di «teorema dell'impossibilità della ridistribuzione» è accettato, la perdita della capacità regolativa nazionale si riduce alla questione relativamente tecnica su dove debbano gravare in prima istanza i costi della (nuova) regolazione ${ }^{34}$. Se essi sono infatti caricati sulle aziende esposte alla competizione internazionale, e se tutte le altre condizioni rimangono identiche, ci sarà allora una caduta della competitività internazionale, ed un concomitante crollo dei profitti, degli investimenti e dell'occupazione. Ma, naturalmente, le altre condizioni non devono necessariamente rimanere le stesse. L'aumento dei costi regolativi potrebbe essere compensato da concessioni salariali, attraverso un aumento della produttività o, finché l'Unione monetaria europea non sarà realizzata, tramite la svalutazione. In effetti, queste misure compensative sposterebbero ancora i costi sulle spalle dei lavoratori e dei consumatori interni.

Tuttavia, lo stesso risultato potrebbe essere ottenuto più direttamente e con una maggiore sicurezza, se già in prima battuta $\mathrm{i}$ costi non fossero riversati sulle aziende. Se le nuove regolazioni sociali, sull'esempio dell'assicurazione tedesca sull'assistenza ai disabili, fossero finanziate attraverso tasse sui redditi e sul consumo, piuttosto che su tasse sui salari, le imprese rimarrebbero competitive e gli investimenti continuerebbero ad essere convenienti. Un esempio è fornito dalla Danimarca, dove l' $85 \%$ dei costi sociali è finanziato da tasse generali. Poiché la competitività internazionale delle imprese danesi non ne è influenzata, il (molto costoso) welfare state non gioca apparentemente nessun ruolo nell'attuale discussione circa la competitività dell'economia nazionale (Münster 1993) ${ }^{35}$. Naturalmente, i

34 Presumibilmente, se un'economia è stata vitale così a lungo, i suoi costi regolativi saranno riflessi nei prezzi correnti e nei tassi di cambio.

35 La più grande minaccia alla salute del modello danese, incidentalmente, viene dal programma europeo di armonizzazione dei livelli dell'IVA. 
redditi disponibili per il consumo saranno ridotti, ma questo è quanto accadrebbe in ogni caso.

Non voglio dire, naturalmente, che tutti gli obiettivi di regolazione sociale dei decenni del dopoguerra potranno essere ottenuti nel futuro senza mettere in pericolo la competitività internazionale. E non sostengo neppure che la crescente resistenza degli elettori nei confronti dell'imposizione fiscale possa essere facilmente sconfitta ${ }^{36}$. A paragone con i primi decenni del dopoguerra, l'ambito delle scelte disponibili per i processi politici democratici al livello nazionale è divenuto certamente molto più basso. Ma non così stretto come il determinismo economico che molti contributi all'attuale dibattito sembrano suggerire. Inoltre, tale ambito di scelta può essere allargato con l'utilizzazione, da parte delle nazioni o delle regioni, dei vantaggi competitivi dovuti alle loro specifiche strutture istituzionali ed industriali al fine di sfruttare delle proprie nicchie nei sempre più specializzati mercati mondiali. Le precondizioni sono, naturalmente, un elevato livello di flessibilità di policy, nonché la capacità di rispondere a specifiche condizioni locali e alle mutevoli occasioni del mercato a tutti i livelli del policy making, europeo, nazionale e subnazionale, così come nelle relazioni industriali e nella gestione del mercato.

L'economia europea ha certamente bisogno di un mercato più largo, ed anche di regole comuni, al fine di poter reggere il confronto con i competitori americani e giapponesi in settori produttivi nei quali le economie di scala fanno una significativa differenza. Ma l'Europa certamente regredirà se l'integrazione negativa dovesse paralizzare la capacità nazionale e sub-nazionale di problem-solving, mentre al livello comunitario potranno essere raggiunti soltanto compromessi insoddisfacenti e dopo lunghe ed estenuanti negoziazioni.

Per aver successo nell'ambito dell'economia globale, l'Europa dipende $\mathrm{da}$ un policy making comunitario più efficace e $\mathrm{da}$ una migliore legittimazione democratica. Ma dipende anche dal mantenimento di un'autonoma capacità di problem-solving $\mathrm{da}$

36 A mio modo di vedere, questa è la vera ragione dell'attuale crisi del welfare state europeo. Dati tassi minori di crescita economica, costi crescenti della protezione ambientale, continua disoccupazione di massa e aumento della quota di popolazione inattiva, la disponibilità degli elettori, facenti parte dei colletti bianchi e dei colletti blu, a sopportare un crescente carico fiscale è divenuto il vincolo critico di tutte le politiche pubbliche che dipendono dalla legittimazione democratica. 
parte dei sistemi politici nazionali e sub-nazionali. Se il dibattito sul principio di sussidiarietà può certo aiutare a limitare il perfezionismo e la rigidità dell'integrazione positiva, abbiamo bisogno anche di un dibattito sull'esigenza di limitare il perfezionismo dell'integrazione negativa. Soltanto se riusciremo a raggiungere entrambi questi obiettivi saremo in grado di combinare l'efficienza economica di un mercato più largo con le capacità di problem-solving di un'azione politica sia a livello europeo che nei sistemi democratici ai livelli nazionali e sub-nazionali.

[Traduzione di Luca Verzichelli]

\section{Riferimenti bibliografici}

Alber, J. e B. Bernardi Schenklunn (1992), Westeuropäische Gesundheitssysteme im Vergleich: Budesrepublik Deutschland, Schweitz, Frankreich, Italien, Großbritannien, Frankfurt, Campus.

Alter, K.J. e S. Meunier-Aitsahalia (1994), Judicial Politics in the European Community. European Integration and the Patbbreaking Cassis de Dijon Decision, in «Comparative Political Studies», 26, pp. 535-561.

Behrens, P. (1994), Die Wirtschaftsverfassung der Europäischen Gemeinschaft, in $\mathrm{G}$. Brüggemeier (a cura di), Verfassungen für ein ziviles Europa, Baden-Baden, Nomos, pp. 73-90.

Burley, A. e W. Mattli (1993), Europe Before the Court: A Political Theory of Legal Integration, in «International Organization», 47, pp. 41-76.

Calhoun, C. (1993), Nationalism and Civil Society: Democracy, Diversity and Self-Determination, in «International Sociology», 8, pp. 387-412.

Crouch, C. (1993), Industrial Relations and European State Traditions, Oxford, Clarendon Press.

Dahl, R.A. (1994), A Democratic Dilemma: System Effectiveness versus Citizen Participation, in «Political Science Quarterly», 109, pp. 23 34.

Dehousse, R. (1993), Does Subsidiarity Really Matter?, Florence, EUI Working Paper Law, n. 92/32.

- (1995), Institutional Reform in the European Community. Are there Alternatives to the Majoritarian Avenue?, Florence, EUI Working Paper RSC, n. 95/4.

Dehousse, R. e J.H.H. Weiler (1990), The Legal Dimension, in W. Wallace (a cura di), The Dynamics of European Integration, London, Pinter, pp. 242-260. 
Eichener, V. (1993), Soziales Dumping oder innovative Regulation? Interessenkonfigurationen und Einflußchancen im Prozeß der Harmonisierung des technischen Arbeitsschutzes, in W. Süß e G. Becher (a cura di), Technologieentwicklung und europäische Integration, Berlin, Duncker u. Humblot, pp. 207-235.

Esping-Andersen, G. (1990), The Three Worlds of Welfare Capitalism, Cambridge, Polity Press.

Featherstone, K. (1994), Jean Monnet and the «Democratic Deficit» in the European Union, in «Journal of Common Market Studies», 132, pp. 149-170.

Fröhlich, T. (1992), Reif für die Mülltonne. Die EG-Verpackungspläne beweisen, daß der Umweltschutz dem Binnenmarkt geopfert werden soll, in «Süddentsche Zeitung», 25-26 luglio, 33.

Ganslandt, H. (1993), Das System der sozialen Sicherung in Griechenland, in G. Lottes (a cura di), Soziale Sicherbeit in Europa. Renten und Sozialversicherungssysteme im Vergleich, Heidelberg, Physica, pp. 185-203.

Garrett, G. (1992), International Cooperation and Institutional Choice: The European Community's Internal Market, in «International Organization», 46, pp. 533-560.

- (1995), The Politics of Legal Integration in the European Union, in «International Organization», 49, pp. 171-181.

Gerhards, J. (1993), Westeuropäische Integration und die Schwierigrkeiten der Entstebung einer europäischen Öffentlichkeit, in «Zeitschrift für Soziologie», 22, pp. 96-110.

Graebner, W. (1977), Federalism in the Progressive Era. A Structural Interpretation of Reform, in «Journal of American History», 64, pp. 331-357.

Grimm, D. (1992), Der Mangel an europäischer Demokratie, in «Der Spiegel», 43/1992, 19 ottobre, pp. 57-59.

Groeben, H. von der (1992), Probleme einer europäischen Wirtschaftsordnung, in J.F. Baur, P.C. Müller-Graff e M. Zuleeg (a cura di), Europarecht, Energierecht, Wirtschaftsrecht. Festschrift für Bodo Barner, Köln, Carl Heymanns, pp. 99-123.

Hank, R. (1994), Die Eisenacher und die Rüsselsheimer. Im Opel-Werk an der Wartburg hat die Zukunft schon begonnen, in «Frankfurter Allgemeine Zeitung», 6 agosto, p. 11.

Heckathorn, D.D. e S.M. Maser (1987), Bargaining and Constitutional Contracts, in «American Journal of Political Science», 31, pp. 142 168.

Héritier, A., S. Mingers, C. Knill e M. Becka (1994), Die Veränderung von Staatlichkeit in Europa. Ein regulativer Wettbewerb, Deutschland, Großbritannien und Frankreich in der Europäischen Union, Opladen, Leske + Budrich.

Joerges, C. (1991), Markt obne Staat? Die Wirtschaftsverfassung der Gemeinschaft und die regulative Politik, in R. Wildenmann (a cura 
di), Staatswerdung Europas? Optionen für eine europäische Union, Baden-Baden, Nomos, pp. 225-268.

- (1994a), Legitimationsprobleme des Europäischen Wiirtschaftsrechts und der Vertrag von Maastricht, in G. Brüggemeier, (a cura di), Verfassungen für ein ziviles Europa, Baden-Baden, Nomos, pp. 91-130.

- (1994b), Rationalisierungsprozesse im Vertragsrecht und im Recht der Produktsicherbeit. Beobachtungen zu den Folgen der Europäischen Integration für das Privatrecht, Florence, EUI Working Paper Law, n. 94/5.

Kapteyn, P. (1991), Civilization under Negotiation. National Civilizations and European Integration: The Treaty of Schengen, in «Archives Europeennes de Sociologie», 32, pp. 363-380.

Kielmansegg, P.G. (1992), Ein Maß für die Größe des Staates. Was wird aus Europa? Europa feblt die Zustimmung seiner Bürger, in «Frankfurter Allgemeine Zeitung», 2 dicembre, p. 35.

Kosonen, P. (1994), The Impact of Economic Integration on National Welfare States in Europe, Manoscritto, University of Helsinki, Department of Sociology.

Lange, P. (1992), The Politics of the Social Dimension, in A.M. Sbragia (a cura di), Euro-Politics; Institutions and Policymaking in the «New» European Community, Washington, D.C., Brookings, pp. 225-256.

Langeheine, B. e U. Weinstock (1984), Graduated Integration: A Modest Path Towards Progress, in «Journal of Common Market Studies», 23, pp. 187-195.

Leibfried, S. e P. Pierson, (1992), Prospects for Social Europe, in «Politics \& Society», 20, pp. 333-366.

Majone, G. (1993), The European Community between Social Policy and Social Regulation, in «Journal of Common Market Studies», 31, pp. 153-170.

- (1994a), The European Community: An Independent Fourth Branch of Government?, in G. Brüggemeier (a cura di), Verfassungen für ein ziviles Europa, Baden-Baden, Nomos, pp. 23-43.

- (1994b), Independence vs. Accountability? Non-Majoritarian Institutions and Democratic Government in Europe, Florence, EUI Working Paper SPS, n. 94/3.

Mattli, W. e A. Slaughter (1995), Law and Politics in the European Union: A Reply to Garrett, in «International Organization», 49, pp. 93-190.

Mayntz, R. e F.W. Scharpf (a cura di) (1995), Steuerung und Selbstorganisation in staatsnaben Sektoren, Frankfurt/M., Campus, in corso di stampa.

Merkel, W. (1993), Ende der Sozialdemokratie? Machtressourcen und Regierungpolitik im westeuropäischen Vergleich, Frankfurt/M., Campus. 
Mestmäker, E.J. (1992), Widersprücblich, verwirrend und gefährlich. Wettbewerbsregeln oder Industriepolitik: Nicht nur in diesem Punkt verstößt der Vertrag von Maastricht gegen bewärte Grundsätze des Vertrages von Rom, in «FAZ», 10.10.12, p. 15.

- (1994), Zur Wirtshaftsverfassung in der Europäischen Union, in R.H. Hasse, J. Molberger e C. Watrin (a cura di), Ordnung in Freibeit. Festgabe für Hans Willgerodt zum 70 Geburrtstag, Stuttgart, Fischer, pp. 263-292.

Müller-Armack, A. (1956), Soziale Marktwirtschaft, in Ders., Wirtschaftsordnung und Wirtschaftpolitik. Studien und Konzepte zur sozialen Marktwirtschaft und zur Europäischen Integration, Freiburg i.b., Rombach, pp. 243-249.

Münster, W. (1993), Vaterstaat shafft es micht mebr. Die Eg im Kampf gegen die Arbeitslosigkeit. Nur neve Märkte Können verlorne Arbeitsplätze Ersetsen, «Süddentsche Zeitung», 23 luglio, p. 31.

Nicoll, W. (1984), Paths to European Unity, in «Journal of Common Markety Studies», 23, pp. 199-206.

Polany, K. (1957), The Great Transformation: the Political and Economic Origin of our Time, Boston, Beacon.

Rehbinder, E. e R. Stewart (1984), Environmental Protection Policy: Integration through Law. Europe and American Federal Experience, Band 2, Berlin, De Gruyter.

Ruggie, J.G. (1982), International Regimes, Transaction and Change: Embedded Liberalism in the Postwar Economic Order, in «International Organization», 36, pp. 379-415.

- (1995), At Home Abroad, Abroad at Home: International Liberalisation and Domestic Stability in the New World Economy.

Sauter, W. (1995), The Telecommunication Laws of the European Community, in «European Law Journal», 1, pp. 92-111.

Scharpf, F.W. (1970), Demokratietheorie zwischen Utopie und Anpassung, Konstanz, Universitätsverlag.

- (1988), The Joint Decision Trap: Lessons from German Federalism and European Integration, in «Public Administration», 66, pp. 239-278.

- (1991a), Crisis and Choice in European Social Democracy, Ithaca, Cornell University Press.

- (1991b), Kann es in Europa eine stabile Föderale Balance geben?, in R. Wildenmann (a cura di), Staatswerdung Europas? Optionen für eine Europäische Union, Baden-Baden, Nomos, pp. 415-428.

- (1992a), Europäisches Demokratiedefizit und deutscher Föderalism, in «Staatswissenschaft und Staatpraxis», 3, pp. 293-306.

- (1992b), Koordination durch Verbandlungssysteme: Analytische Konzepte und institutionelle Lösungen, in A. Benz, Fritz W. Scharpf e R. Zintl, Horizontale Politikverflechtung. Zur Theorie von Verhandlungssystemen, Frankfurt/M., Campus, pp. 51-96.

- (1993), Versuch über Demokratie im verbandelnden Staat, in R. 
Czada e M.G. Schmidt (a cura di), Verhandlungsdemokratie, Interessenvermittlung, Regierbarkeit. Festscrift für Gerhard Lehmbruch, Opeaden, Westdeutscher Verlag, pp. 25-50.

- (1994), Community and Autonomy: Multilevel Policy-making in the European Union, in «Journal of European Public Policy», 1, pp. 219-242.

Schmidt, S.K. (1995), The Integration of the European Telecommunications and Electricity Sectors in the Light of International Relations Theories and Comparative Politics, manoscritto, Köln, Max-Planck-Institut für Gesellshaftsforschung.

Schneider, V. (1995), Institutionelle Evolution als politischer Prozeß: Die Entwicklung der Telekommunikation im historischen und internationalen Vergleich, manoscritto, Köln, Max-Planck-Institut für Gesellschaftsforschung.

Schwartz, B. (1957), The Supreme Court. Constitutional Revolution in Retrospect, New York, Ronald Press.

Sieber, W. (1993), Die soziale Sicherung in Portugal vor dem Hintergrund von EG-Integration und beschleunigtem wirtschaftlich-sozialem Strukturwandel, in G. Lottes (a cura di), Soziale Sicherbeit in Europa. Renten- und Sozialversicherungssysteme im Vergleich, Heidelberg, Physica, pp. 171-185.

Sinn, H.W. (1994), Wieviel Brüssel braucht Europa? Subsidiarität, Zentralisierung und Fiskalwettbewerb im Lichte der ökonomischen Theorie, in «Staatswissenschaften und Staatspraxis», 5, pp. 155186.

Sinn, S. (1993), The Taming of Leviathan. Competition among Governments, in «Constitutional Political Economy», 3, pp. 177-221.

Streeck, W. (1993), From Market Making to State Building? Reflections on the Political Economy of European Social Policy, manoscritto, Madison, University of Wisconsin.

Streit, M.E. e W. Mussler (1995), The Economic Constitution of the European Community: From «Rome» to «Maastricht», in «European Law Journal», 1, pp. 5-30.

Tinbergen, J. (1965), International Economic Integration, Second Edition, Amsterdam, Elsevier.

Tsebelis, G. (1995), Decision making in Political Systems: Veto Players in Presidentialism, Parliamentarism, Multicameralism, and Multipartism, in corso di pubblicazione su «British Journal of Political Science».

Verloren van Themaat, P. (1987), Die Aufgabenverteilung zwischen dem Gesetzgeber und dem Europäischen Gerichtshof bei der Gestaltung der Wirtschaftsverfassung der Europäischen Gemeinschaften, in E.J. Mestmacker, H. Möller e H.P. Schwarz (a cura di), Eine Ordnungspolitik für Europa. Festschrift für Hans von der Groeben, Baden-Baden, Nomos, pp. 425-443.

Voelzkow, H. (1993), Staatseingriff und Verbandsfunktion: Das verban- 
dliche System technischer Regelsetzung als Gegenstand staatlicher Politik, Discussion Paper 93/2, Köln, Max-Planck-Institut für Gesellschaftsforschung.

Wallerstein, M. (1990), Class Conflict as a Dynamic Game, in R. Friedland e A.F. Robertson (a cura di), Beyond the Market-place. Retbinking Economy and Society, New York, Aldine de Gruyter, pp. 189-212.

Weidenfeld, W. (1995), Europe '96. Reforming the European Union, Gütersloh, Bertelsmann Foundation Publishers.

Weiler, Joseph H.H. (1981), The Community System. The Dual Character of Supranationalism, in «Yearbook of European Law», 1, pp. 257-306.

Weiler, J.H.H. (1993), Journey to an Unknown Destination: A Retrospective and Prospective of the European Court of Justice in the Arena of Political Integration, in «Journal of Common Market Studies», 31, pp. 417-446.

- (1994), A Quiet Revolution. The European Court of Justice and Its Interlocutors, in «Comparative Political Studies», 26, pp. 510-534.

Weizsäcker, E.U. von (1989), Internationale Harmonisierung im Umneltschutz durch Ökonomische Instrumente - Gründe für eine europäische Umweltsteuer, in «Jahrbuch zur Staats- und Verwaltungswissenschaft», Band 3/1989, pp. 203-216.

Williams, S. (1991), Sovereignty and Accountability in the European Community, in R.O. Keohane e S. Hoffmann, (a cura di), The New European Community. Decision Making and Institutional Change, Boulder, Westview, pp. 155-176.

Wilson, T.M. e M.E. Smith (a cura di) (1993), Cultural Changes and the New Europe: Perspectives on the European Community, Boulder, Westview. 